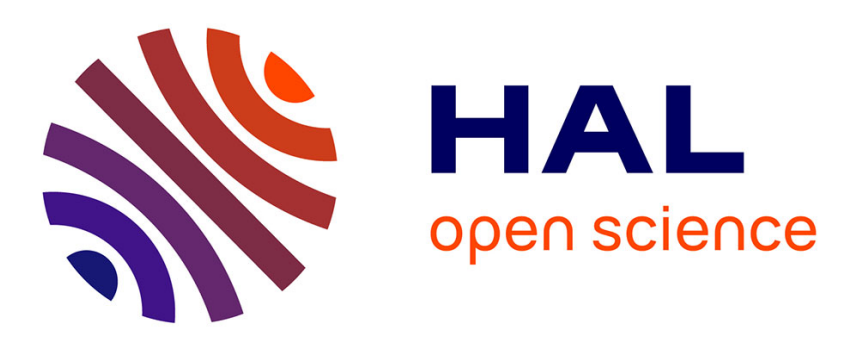

\title{
Three-dimensional constitutive model for structural and functional fatigue of shape memory alloy actuators
}

\author{
Yves Chemisky, Darren J. Hartl, Fodil Meraghni
}

\section{To cite this version:}

Yves Chemisky, Darren J. Hartl, Fodil Meraghni. Three-dimensional constitutive model for structural and functional fatigue of shape memory alloy actuators. International Journal of Fatigue, 2018, 112, pp.263-278. 10.1016/j.ijfatigue.2018.03.016 . hal-01762224

\section{HAL Id: hal-01762224 \\ https://hal.science/hal-01762224}

Submitted on 9 Apr 2018

HAL is a multi-disciplinary open access archive for the deposit and dissemination of scientific research documents, whether they are published or not. The documents may come from teaching and research institutions in France or abroad, or from public or private research centers.
L'archive ouverte pluridisciplinaire HAL, est destinée au dépôt et à la diffusion de documents scientifiques de niveau recherche, publiés ou non, émanant des établissements d'enseignement et de recherche français ou étrangers, des laboratoires publics ou privés. 


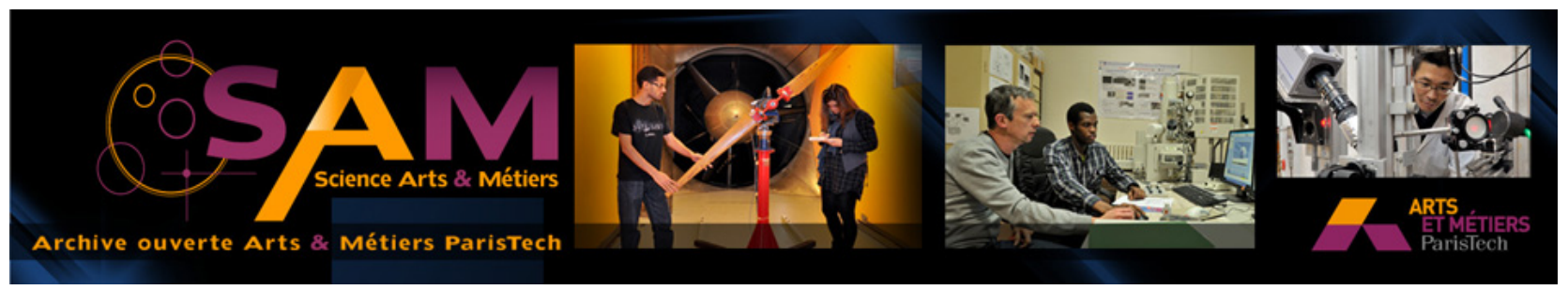

Science Arts \& Métiers (SAM)

is an open access repository that collects the work of Arts et Métiers ParisTech researchers and makes it freely available over the web where possible.

This is an author-deposited version published in: http://sam.ensam.eu

Handle ID: .http://hdl.handle.net/null

\section{To cite this version :}

Yves CHEMISKY, Darren J. HARTL, Fodil MERAGHNI - Three-dimensional constitutive model for structural and functional fatigue of shape memory alloy actuators - International Journal of Fatigue p.in press -2018 


\title{
Three-dimensional constitutive model for structural and functional fatigue of shape memory alloy actuators
}

\author{
Yves Chemisky $^{\mathrm{a}, *}$, Darren J. Hartl ${ }^{\mathrm{b}}$, Fodil Meraghni ${ }^{\mathrm{a}}$ \\ ${ }^{a}$ Arts et Métiers ParisTech, Laboratoire d'étude des Microstructures et de Mécanique des Matériaux \\ (LEM3) CNRS, 57078 Metz, France \\ ${ }^{b}$ Department of Aerospace Engineering, \\ Texas A\&M University, College Station, TX 77843-3409, USA
}

\begin{abstract}
A three-dimensional constitutive model is developed that describes the behavior of shape memory alloy actuators undergoing a large number of cycles leading to the development of internal damage and eventual catastrophic failure. Physical mechanisms such as transformation strain generation and recovery, transformation-induced plasticity, and fatigue damage associated with martensitic phase transformation occurring during cyclic loading are all considered within a thermodynamically consistent framework. Fatigue damage in particular is described utilizing a continuum theory of damage. The total damage growth rate has been formulated as a function of the current stress state and the rate of martensitic transformation such that the magnitude of recoverable transformation strain and the complete or partial nature of the transformation cycles impact the total cyclic life as per experimental observations. Simulation results from the model developed are compared to uniaxial actuation fatigue tests at different applied stress levels. It is shown that both lifetime and the evolution of irrecoverable strain are accurately predicted by the developed model.
\end{abstract}

\section{Introduction}

Shape memory alloys (SMAs) are metals that have the ability to generate and recover substantial deformation during a thermomechanical cycle. The physical mechanism that drives the shape recovery in the materials is a martensitic phase transformation that results from thermal and/or mechanical inputs, often without the consequence of significant plastic

\footnotetext{
${ }^{*}$ Corresponding author.

Email address: yves.chemisky@ensam.eu (Yves Chemisky)
} 
strain generation during formation and recovery of martensitic variants. This unique ability has led to the development of devices for aerospace and medical applications (Hartl and Lagoudas, 2007; Mohd Jani et al., 2014; Lester et al., 2015). The design of such devices has required the development of constitutive models to predict their thermomechanical behavior. A comprehensive review of SMA constitutive models can be found in works by Birman (1997), Patoor et al. (2006), Lagoudas et al. (2006), Paiva et al. (2006), and Cisse et al. (2015).

Early models describe the behavior of conventional SMAs without considering irrecoverable strains and damage, which is sufficient for the design of devices where operating temperatures, maximum stress levels, and number of actuation cycles are all relatively low. To expand the capabilities of such models, the evolution of transformation induced plasticity was first considered for conventional SMAs by Bo and Lagoudas (1999b) and then Lagoudas and Entchev (2004); these models allow calculations of accumulated irrecoverable strains caused by cycling. The coupling between phase transformation and plasticity at higher stresses has been considered in the literature for the simulation of shape memory alloy bodies under high loads at low temperatures compared to their melting points (Hartl and Lagoudas, 2009; Zaki et al., 2010; Khalil et al., 2012). A model accounting for the effect of retained martensite (martensite pinned by dislocations) has been developed by Saint-Sulpice et al. (2009). To predict the influence of irrecoverable strains in high-temperature SMAs (HTSMAs) where viscoplastic creep is observed, a one-dimensional model accounting for the coupling between phase transformation and viscoplasticity has been developed by Lagoudas et al. (2009a); a three-dimensional extension of this model was developed and implemented via finite element analyses (FEA) by Hartl et al. (2010b), and the cyclic evolution of irrecoverable strains accounting for combined viscoplastic, retained martensite, and TRIP effects was later implemented by Chemisky et al. (2014). blueThe evolution of the pseudoelastic response for low cycle fatigue of SMAs has been invesigated recently (Zhang et al., 2017). A strain-energy based fatigue model has been proposed and confronted to experiments.

These past efforts focused on the prediction of thermomechanical responses for only a small number of cycles (e.g., up to response stabilization). However, active material actuators are often subjected to a large number of repeated cycles (Van Humbeeck, 1999; Mohd Jani et al., 2014), which induces thermally-induced fatigue in the case of SMAs (Lagoudas 
et al., 2000; Bertacchini et al., 2009). During the lifetime of an SMA actuator, the influence of two different classes of fatigue must be considered:(i) Structural fatigue is the phenomenon that leads towards catastrophic failure of components, while (ii) functional fatigue describes permanent geometric changes to the detriment SMA of component performance and is associated with the development of irrecoverable strain( Eggeler et al. (2004)). The prediction of functional fatigue evolution allows for calculation of changes expected in a given actuator over its lifetime, while the prediction of structural fatigue evolution allows for determination of the actuator lifetime itself.

While the prediction of functional fatigue relies on the simulation of irrecoverable strains upon cycling (i.e., so-called trans-induced plasticity(TRIP)) (Bo and Lagoudas, 1999b; Lagoudas and Entchev, 2004), catastrophic structural fatigue is associated with the development of micro-cracks during transformation. Most SMAs are herein taken to be sufficiently similar to hardening metal materials, so as to apply the theoretical modeling of structural fatigue via thermodynamic approaches developed in recent years. (Khandelwal and Buravalla, 2009). Continuum damage mechanics (CDM) has been extensively utilized to predict the fatigue lifetime of metallic materials and structures since its development and integration within the framework of thermodynamics of irreversible processes (Bhattacharya and Ellingwood, 1998; Lemaitre and Desmorat, 2005; Dattoma et al., 2006). The notion of damage itself concerns the progressive degradation of the mechanical properties of materials before the initiation of cracks observable at the macro-scale (Simo and Ju, 1987).

Contrary to approaches based on fracture mechanics, which explicitly consider the initiation and growth of micro-cracks, voids, and cavities as a discontinuous and discrete phenomenon (Baxevanis and Lagoudas, 2015), CDM describes damage using a continuous variable associated with the local density of micro-defects. Based on this damage variable, constitutive equations have been developed to predict the deterioration of material properties (Voyiadjis and Kattan, 1999). CDM enables fatigue life prediction in innovative superalloys (Shi et al., 2013) and standard aluminium alloys (Hojjati-Talemi and Wahab, 2013) alike. Relevant models can also be implemented within FEA framework to predict the response of structures with complex shapes (Zhang et al., 2012). Two opposing views exist in the theoretical modeling of continuous damage. If the micro-defects and their associated effects 
are considered isotropic, a simple scalar variable (i.e.,the damage accumulation) is sufficient to describe the impact of damage on material properties. However, to comply with experimental findings confirming anisotropic evolution of damage in ductile materials (Lemaitre et al., 2000; Bonora et al., 2005; Luo et al., 2012; Roth and Mohr, 2014), researchers have also developed anisotropic damage continuum models as proposed by Voyiadjis and Deliktas (2000); Brünig (2003); Desrumaux et al. (2001). In this latter case, the distribution of micro-defects adopts preferred orientations throughout the medium. To model this behavior, a tensorial damage variable is typically introduced, (i.e., the damage effect tensor) (Lemaitre et al., 2000; Lemaitre and Desmorat, 2005). A set of internal variables that are characteristic of various damage mechanisms can also be considered (Ladeveze et al., 1992; Mahboob et al., 2017).

CDM models are also categorized based on the mathematical approach utilized. Strictly analytical formalisms belong to the group of deterministic approaches. These utilize robust thermodynamic principles, thermodynamic driving forces, and a critical stress threshold to derive mathematical expressions linking the damage variable with the material properties and other descriptions of state. The appearance of micro-defects below such stress thresholds is not considered possible and every result represents a deterministic prediction of material behavior. Alternatively, probabilistic approaches define probabilities attributed to the appearance of micro-defects. The damage is often thought to occur at points in the material where the local ultimate strength is lower than the average stress. Considering the local ultimate stress as a stochastic variable leads to calculated damage evolution that is likewise probabilistic. Such probability can be introduced into a thermodynamic model that describes the material properties to within margins of error (Fedelich, 1998; Rupil et al., 2011).

The probabilistic models have been built mostly to treat fracture in brittle materials, such as ceramics (Hild et al., 2003) or cement (Grasa et al., 2005), which demonstrate statistical scatter in direct relation with damage such as crack initiation and coalescence (Meraghni et al., 2002). Probabilistic modeling may be a useful tool in fatigue life analysis of SMA bodies, given the scattering observed in the thermomechanical response of nearly identical test samples demonstrated in experimental works (Figueiredo et al., 2009; Nemat-Nasser and Guo, 2006). Relevant experiments (Scirè Mammano and Dragoni, 2014) determine the 
number of cycles to failure in NiTi SMA wires by considering samples submitted to a series of cyclic load fatigue tests at increasing strain rates. It is evident from such works that the fatigue life is to some degree uncertain and the use of stochastic models might increase prediction accuracy overall.

Several fatigue failure models for SMAs have been developed based on experimental observations. Tobushi et al. (2000) have proposed an empirical fatigue life equation, similar to a Coffin-Manson law, that depends on strain amplitude, temperature, and frequency of the cycles. This first model was compared to rotating-bending fatigue tests. A modified Manson-Coffin model was further proposed by Maletta et al. (2012, 2014) to predict the fatigue life of NiTi SMAs under the stress-controlled cyclic loading conditions. A third Manson Coffin-like relationship has been proposed by Lagoudas et al. (2009b) to determine the irrecoverable strain accumulation of NiTiCu SMAs as a function of the number of cycles to failure for different stress levels, for both partial and complete transformations.

Energy-based fatigue life models for SMAs have also been developed, and in particular consider the dissipated energy. Moumni et al. (2005) proposed an empirical power law to predict the fatigue life of super elastic NiTi SMAs. Kan et al. (2012) has modified the previous model, replacing the power-law equation by a logarithmic one. Those models were compared with fatigue tests performed on NiTi alloys under uniaxial stress-controlled cyclic loading (Kang et al., 2012).

Song et al. (2015) has recently proposed a damage-based fatigue failure model, considering three damage mechanisms, (i.e. micro-crack initiation, micro-crack propagation, and martensite transformation induced damage). A global damage variable is defined as the ratio of the accumulated dissipation energy at the current number of cycles $(N)$ with regard to the accumulated dissipation energy obtained at the failure life $\left(N_{f}\right)$. A damage-based fatigue failure model is proposed to predict the fatigue life, that depends on the dissipation energy at the stabilized cycle, and the dissipation energy at the N-th cycle. It is shown that the model predicts the fatigue life of super-elastic NiTi SMA micro-tubes subjected to uniaxial stress-controlled load cycles. blueHigh cycle fatigue criterion have been developed recently for SMAs. The Investigation of SMA cyclic response under elastic shakedown has led to the definition of a Dang Van, which means type endurance limit for SMA materials (Auricchio 
et al., 2016). A Shakedown based model for high-cycle fatigue of shape memory alloys has been developed by $\mathrm{Gu}$ et al. (2016). Non-proportional multiaxial fatigue of pseudoelastic SMAs has been recently investigated by Song et al. (2017), which has led to the definition of a multiaxial fatigue model.

Although past developments allow determination of the fatigue life of shape memory alloy devices for uniaxial, homogeneous cyclic loadings, the present work focuses on the difficult problem of coupling damage evolution to phase transformation, irrecoverable transformationinduced plastic strain, and general three-dimensional thermomechanical states. To permit the introduction of damage into a previously well-defined and widely accepted class of model for SMA phase transformation ( Lagoudas et al. (2012)), probabilistic and anisotropic approaches are avoided. Rather, a deterministic and isotropic model for continuum damage mechanics is proposed, which is compatible with the existing models of thermomechanical response of SMA actuators, including those considering generated plastic strain consideration herein ( Chemisky et al. (2014)). Such a model, even once its assumptions are considered, provides the most comprehensive tool for calculating fatigue in SMA actuators to date.

The organization of this work follows. The motivation of the proposed model, including the need for numerical simulations of cyclic loading in SMA bodies, has been provided in Section 1. Observations motivating specific forms of the evolution equations for damage and irrecoverable strains are overviewed in Section 2. The thermodynamical model is developed in Section 3, with the functional form of the various evolution equations related to the physical mechanisms considered being clearly presented. After some comments on model calibration in Section 4, numerical simulations and their comparison with experimental demonstrations of structural and functional fatigue in SMA bodies are presented in Section 5. Final conclusions are provided in Section 6.

\section{Motivating Observations from Previous Studies}

Studies of SMA actuation fatigue are not as numerous as those focusing on nearly isothermal (i.e., superelastic) response. This is due to both the relative importance of generally isothermal medical devices and the difficulty of applying high numbers of thermal cycles to SMA actuators. From those SMA actuation fatigue databases that are available in the 
literature, the experimental studies of actuation fatigue and post-mortem analyses that were carried out on Ni60Ti40 (wt. \%) (Agboola et al., 2012) and on NiTiHf (Wheeler et al., 2015) have been selected for consideration herein. In those past studies, a widespread distribution of cracks were found to be present in the SMA components at failure, as shown in Fig. 1. This indicates that a progressive damage mechanism was activated during the lifetime of the SMA body. CDM appears to be particularly adapted for modeling such fatigue damage given the continuous and progressive evolution of multiple defects observed.

NiTiHf alloys have received an increased attention in the recent years according to their high potential to be utilized for high temperature actuators, (e.g., those having transformation temperatures typically above $100^{\circ} \mathrm{C}$ ). They can operate at high stress levels without the development of significant plastic strains (Karaca et al., 2014). From the analysis of NiTiHf actuator fatigue tests, we see that the number of cycles to failure increases with decreasing cyclic actuation work. Actuator work is defined as the scalar product of the constant applied stress and the transverse strain recovered each cycle (see Fig. 2a). Further, the amount of irrecoverable strain generated seems to be positively correlated with the number of cycles to failure (see Fig. 2b). The development of such strains may be important in predicting the lifetime of actuators formed from a number of SMA materials or some stress levels, bluespecifically at higher stress levels (e.g., 300-600 MPa in (Wheeler et al., 2015)). The study of fatigue in Ni60Ti40 actuator components also shows that the number of cycles to failure increases with decreasing cyclic actuation work (see Fig. 2c). However, the experimental results suggests that in this material loaded to lower stresses blue(e.g., 100-250 MPa in (Agboola et al., 2012)), failure may not be correlated with the accumulation of plastic strain, though such correlation is regularly considered in ductile metals. This is shown for the lower stressed Ni60Ti40 samples (Fig. 2d). The consistent and clear negative correlation between cyclic actuation work and fatigue life in both cases motivates the choice of a thermodynamical model to describe the evolution of damage in shape memory alloys. The fact that generated TRIP might also be correlated to failure at higher stresses only motivates consideration of a stress threshold effect for the coupling between damage and TRIP strains, and this will be addressed for the first time herein. 


\section{Constitutive Modeling Framework for Phase Transformation and Damage}

Physical mechanisms associated with cyclic martensitic phase transformation such as transformation strain generation and recovery, transformation-induced plastic strain generation, and fatigue damage accumulation are all taken into account within the thermodynamically consistent constant model presented in this section. Fatigue damage is described utilizing a scalar variable following an isotropic continuum theory of damage. The damage growth rate has been formulated as a function of both the stress state and the magnitude of the recoverable transformation strain such that cyclic actuation work is directly and clearly considered. Transformation-induced plasticity is also considered as per the experimental observations described in the previous section, and its generation depends on the stress state, the magnitude of the transformation strain, and a term that couples with plastic strain with damage for stress levels above an assumed material-dependent threshold.

The model is based on the framework of Lagoudas et al. (2012), considering further improvements proposed by Chatziathanasiou et al. (2016) for thermodynamical models describing phase transformation that drives multiple complex phenomena. The proposed model focuses on the generation and recovery of transformation strains that occur as a result of martensitic transformation (forward and reverse); martensitic reorientation is not considered

Figure 1: Examples of damage (micro-crack) in nickel-rich NiTi material after thermally-induced actuation cycling (2840 cycles at 200MPa). Note the micro-cracks initiating within precipitates, resulting in relative small observable strains but eventually leading to specimen failure (Agboola et al., 2012)

Figure 2: Indications of structural and functional fatigue for two classes of SMA materials. a) and c): Structural fatigue in Ni60Ti40 and NiTiHf actuators, respectively, where a strong correlation between actuation work and cycles to failure is observed in each. b) and d): Functional fatigue of Ni60Ti40 and NiTiHf actuators, respectively, where correlation between number of cycles to failure and the level of irrecoverable strains depends on the level of stress and alloy. Data for Ni60Ti40 taken from Agboola et al. (2012). Data for NiTiHf taken from Wheeler et al. (2015). 
given its relative unimportance in SMA cyclic actuator applications, which are obviously the primary motivating application for this work. Concerning damage evolution, it is herein assumed, based on observations, that microscopic crack initiation and propagation are not explicitly linked to the appearance of large plastic strains (Bertacchini et al., 2009; Calhoun et al., 2015), but rather that the process of martensitic phase transformation may be more important. In fact, it has been shown the localized nucleation of martensite around crack tips during forward transformation can decrease the fracture toughness and induce localized propagation of cracks, even under moderate stresses (Baxevanis and Lagoudas, 2015). The evolution of damage must therefore be coupled with the martensitic transformation mechanisms directly. The framework thus adopted follows closely the work of Lagoudas and Entchev (2004) and Chemisky et al. (2014) for the development of TRIP strain and Lemaitre and Chaboche (2002) for the coupling between a physical mechanism such as plasticity or phase transformation with damage.

To summarize, the following internal state variables associated with multiple inelastic strain mechanisms are tracked during both forward and reverse transformations:

- The inelastic transformation strain $\varepsilon^{\mathrm{t}}$, which considers all inelastic strains associated with different physical phenomena occurring during transformation (i.e, it is composed of contributions from crystallographic transformation, plasticity, and damage). Such transformation strain is decomposed in two contributions, $\varepsilon^{\mathrm{F}}$ and $\varepsilon^{\mathrm{R}}$, to represent the inelastic strain induced by forward transformation and by reverse transformation, respectively. The inelastic transformation strain is further split into a part that is recoverable(tt) and a portion that is not (TRIP strain;tp), to obtain four total contributions : $\varepsilon^{\mathrm{tt}-\mathrm{F}}, \varepsilon^{\mathrm{tp}-\mathrm{F}}, \varepsilon^{\mathrm{tt}-\mathrm{R}}$ and $\varepsilon^{\mathrm{tp}-\mathrm{R}}$, such that:

$$
\varepsilon^{\mathrm{t}}=\varepsilon^{\mathrm{F}}+\varepsilon^{\mathrm{R}}=\varepsilon^{\mathrm{tt}-\mathrm{F}}+\varepsilon^{\mathrm{tp}-\mathrm{F}}+\varepsilon^{\mathrm{tt}-\mathrm{R}}+\varepsilon^{\mathrm{tp}-\mathrm{R}}
$$

- The scalar total martensitic volume fractions induced by forward transformation (into martensite) and by reverse transformation (into austenite) $\left(\xi^{\mathrm{F}}, \xi^{\mathrm{R}}\right)$,

- The scalar transformation hardening energies induced by forward transformation and by reverse transformation $\left(g^{\mathrm{F}}, g^{\mathrm{R}}\right)$, 
- The scalar accumulated transformation-induced plastic strain accompanying forward transformation and reverse transformation $\left(p^{\mathrm{F}}, p^{\mathrm{R}}\right)$,

- The scalar plastic hardening energy induced by forward transformation and reverse transformation $\left(g^{\mathrm{tp}-\mathrm{F}}, g^{\mathrm{tp}-\mathrm{R}}\right)$,

- The scalar (i.e., isotropic) damage accumulation induced during forward transformation and reverse transformation $\left(d^{\mathrm{F}}, d^{\mathrm{R}}\right)$.

Considering the point-wise model as describing a representative volume element of volume $V$ (Bo and Lagoudas, 1999a) and acknowledging that both forward and reverse transformations can occur simultaneously at various points within such a finite volume, the following two rate variables are introduced : (i) $\dot{\xi}^{\mathrm{F}}$ represents the fractional rate of change of the martensitic volume $V_{M}$ induced by forward transformation (Chatziathanasiou et al., 2016):

$$
\dot{\xi}^{\mathrm{F}}=\frac{\dot{V}_{\mathrm{M}}^{\mathrm{F}}}{V} .
$$

Similarly,(ii) $\dot{\xi}^{\mathrm{R}}$ represents the rate of change of the martensitic volume fraction (MVF) induced by reverse transformation:

blue

$$
\dot{\xi}^{\mathrm{R}}=-\frac{\dot{V}_{\mathrm{M}}^{\mathrm{R}}}{V} .
$$

The rate of the total martensitic volume fraction $\dot{\xi}$ is then:

$$
\dot{\xi}=\dot{\xi}^{\mathrm{F}}-\dot{\xi}^{\mathrm{R}}
$$

which leads to the definition of the total volume fraction of martensite:

$$
\xi=\int_{0}^{t} \dot{\xi}^{F} d \tau-\int_{0}^{t} \dot{\xi}^{R} d \tau=\xi^{\mathrm{F}}-\xi^{\mathrm{R}} .
$$

blueNote that $\xi^{\mathrm{F}}$ and $\xi^{\mathrm{R}}$ always take positive values, which simplifies the thermodynamic definition of the model. The physical limitation related to the definition of total volume fraction is expressed as:

$$
0 \leq \xi \leq 1 \quad \Leftrightarrow \quad \xi^{\mathrm{R}} \leq \xi^{\mathrm{F}} \leq 1+\xi^{\mathrm{R}}
$$


Similarly, the rates of the various strain measures of the total accumulated plastic strain $p$, the total transformation hardening energy $g^{\mathrm{t}}$, the total plastic hardening $g^{\mathrm{tp}}$, and the total damage $d$ are taken to be the sums of contributions from both forward and reverse transformations:

$$
\begin{aligned}
\dot{\varepsilon}^{\mathrm{t}-\mathrm{F}} & =\dot{\varepsilon}^{\mathrm{tt}-\mathrm{F}}+\dot{\varepsilon}^{\mathrm{tp}-\mathrm{F}} \\
\dot{\varepsilon}^{\mathrm{t}-\mathrm{R}} & =\dot{\varepsilon}^{\mathrm{tt}-\mathrm{R}}+\dot{\varepsilon}^{\mathrm{tp}-\mathrm{R}} \\
\dot{\varepsilon}^{\mathrm{tt}} & =\dot{\varepsilon}^{\mathrm{tt}-\mathrm{F}}+\dot{\varepsilon}^{\mathrm{tt}-\mathrm{R}} \\
\dot{\varepsilon}^{\mathrm{tp}} & =\dot{\varepsilon}^{\mathrm{tp}-\mathrm{F}}+\dot{\varepsilon}^{\mathrm{tp}-\mathrm{R}} \\
\dot{\xi} & =\dot{\xi}^{\mathrm{F}}-\dot{\xi}^{\mathrm{R}} \\
\dot{g}^{\mathrm{t}} & =\dot{g}^{\mathrm{F}}+\dot{g}^{\mathrm{R}} \\
\dot{p} & =\dot{p}^{\mathrm{F}}+\dot{p}^{\mathrm{R}} \\
\dot{g}^{\mathrm{tp}} & =\dot{g}^{\mathrm{tp}-\mathrm{F}}+\dot{g}^{\mathrm{tp}-\mathrm{R}} \\
\dot{d} & =\dot{d}^{\mathrm{F}}+\dot{d}^{\mathrm{R}} .
\end{aligned}
$$

In this way, two sets of internal variables respectively related to forward transformation (into martensite) and to reverse transformation (into austenite) are defined:

$$
\begin{aligned}
\boldsymbol{\zeta}^{\mathrm{F}} & =\left\{\xi^{\mathrm{F}}, \boldsymbol{\varepsilon}^{\mathrm{tt}-\mathrm{F}}, \boldsymbol{\varepsilon}^{\mathrm{tp}-\mathrm{F}}, g^{\mathrm{F}}, p^{\mathrm{F}}, g^{\mathrm{tp}-\mathrm{F}}, d^{\mathrm{F}}\right\} \\
\boldsymbol{\zeta}^{\mathrm{R}} & =\left\{\xi^{\mathrm{R}}, \boldsymbol{\varepsilon}^{\mathrm{tt}-\mathrm{R}}, \boldsymbol{\varepsilon}^{\mathrm{tp}-\mathrm{R}}, g^{\mathrm{R}}, p^{\mathrm{R}}, g^{\mathrm{tp}-\mathrm{R}}, d^{\mathrm{R}}\right\} \\
\boldsymbol{\zeta} & =\left\{\boldsymbol{\zeta}^{\mathrm{F}}, \boldsymbol{\zeta}^{\mathrm{R}}\right\}
\end{aligned}
$$

blueTo rigorously derive a three-dimensional model for damage accumulation in SMA materials that explicitly couples actuation work to material degradation, the thermodynamics of irreversible processes are utilized. The fundamentals are presented in Annex A.

\subsection{Thermodynamic derivation of the proposed model}

The total Gibbs free energy $\mathrm{G}$ is additively decomposed into a thermoelastic contribution

$G^{A}$ from regions of the RVE in the austenitic phase, a thermoelastic contribution $G^{M}$ from regions of the RVE in the martensitic phase, and a mixing term $G^{\text {in }}$ that accounts for 
non-thermoelastic processes: Given the state variables chosen for the description of the thermomechanical mechanisms, the Gibbs energy for the overall SMA material is written:

$$
G=(1-\xi) G^{A}(\boldsymbol{\sigma}, \theta, d)+\xi G^{M}(\boldsymbol{\sigma}, \theta, d)+G^{\mathrm{mix}}\left(\boldsymbol{\sigma}, \boldsymbol{\varepsilon}^{\mathrm{tt}}, g^{\mathrm{t}}\right)
$$

The part of the Gibbs free energy related to the martensitic transformation only is taken from the model of Lagoudas et al. (2012) given the conventional dependence of elastic response on damage (Lemaitre and Desmorat, 2005), such that

$$
\begin{aligned}
G^{\beta}(\boldsymbol{\sigma}, \theta, d) & =-\frac{1}{2(1-d)} \boldsymbol{\sigma}: \mathcal{S}^{\beta}: \boldsymbol{\sigma}-\boldsymbol{\sigma}: \boldsymbol{\alpha}\left(\theta-\theta_{0}\right) \\
& +c^{\beta}\left[\left(\theta-\theta_{0}\right)-\theta \ln \left(\frac{\theta}{\theta_{0}}\right)\right]-\eta_{0}^{\beta} \theta+E_{0}^{\beta}
\end{aligned}
$$

for $\beta=A, M$.

Whereas the energy of phase mixing is given as:

$$
G^{\mathrm{mix}}\left(\boldsymbol{\sigma}, \boldsymbol{\varepsilon}^{\mathrm{t}}, g^{\mathrm{t}}, g^{\mathrm{tp}}\right)=\boldsymbol{\sigma}: \boldsymbol{\varepsilon}^{\mathrm{t}}+g^{\mathrm{t}}+g^{\mathrm{tp}}
$$

In those expressions above, $\mathcal{S}$ is the compliance tensor (4th order), $\boldsymbol{\alpha}$ is the thermal expansion tensor (2nd order), $c_{0}$ is a material parameter that approximates as the specific heat capacity (additional terms arising from thermo-inelastic coupling being small (Rosakis et al. $(2000))), \eta_{0}$ is the initial entropy, $E_{0}$ is the initial internal energy, and $\theta_{0}$ is the initial or reference temperature. Details about the selection of the thermoelastic contribution of the phases, especially considering the term related to heat capacity, are given in Chatzigeorgiou et al. (2018). It is assumed, (Lagoudas et al., 2012; Chemisky et al., 2014; Chatziathanasiou et al., 2016), that thermoelastic parameters (including specific heat) that enter the expression of the Gibbs free energy for each phase can be regrouped into phase-dependent parameters as experiments warrant (i.e., $\mathcal{S}(\xi), \boldsymbol{\alpha}(\xi), c(\xi), \eta_{0}(\xi)$ and $\left.E_{0}(\xi)\right)$, where a linear rule of mixtures is assumed. For example, $\mathcal{S}(\xi)$ is the linearly phase-dependent and, written as

$$
\mathcal{S}(\xi)=\mathcal{S}^{\mathrm{A}}-\xi\left(\mathcal{S}^{\mathrm{A}}-\mathcal{S}^{\mathrm{M}}\right)=\mathcal{S}^{\mathrm{A}}-\xi \Delta \mathcal{S}
$$

where $\mathcal{S}^{\mathrm{A}}$ and $\mathcal{S}^{\mathrm{M}}$ denote the compliance tensors of austenite and martensite, respectfully, and the operator $\Delta$ denotes the difference in any material constant as measured in the 
pure martensite and pure austenite phases. Conventionally, standard isotropic forms are assumed sufficient for $\mathcal{S}$ and $\boldsymbol{\alpha}$ in polycrystals. Recalling that the transformation strain $\varepsilon^{t}$ includes all deformations associated with martensitic transformation, recoverable and irrecoverable, the following thermodynamical quantities are expressed, recalling the method proposed by Germain et al. (1983) and invoking (8), (A.15), and (10):

$$
\begin{aligned}
\boldsymbol{\varepsilon} & =-\frac{\partial G}{\partial \boldsymbol{\sigma}}=\frac{1}{1-d} \mathcal{S}: \boldsymbol{\sigma}+\boldsymbol{\alpha}\left(\theta-\theta_{0}\right)+\boldsymbol{\varepsilon}^{\mathrm{t}} \\
\eta & =-\frac{\partial G}{\partial \theta}=\boldsymbol{\alpha}: \boldsymbol{\sigma}+c_{0} \ln \left(\frac{\theta}{\theta_{0}}\right)+\eta_{0}, \\
\gamma_{\mathrm{loc}} & =-\frac{\partial G}{\partial \boldsymbol{\varepsilon}^{\mathrm{t}}}: \dot{\boldsymbol{\varepsilon}}^{\mathrm{t}}-\frac{\partial G}{\partial g^{\mathrm{t}}} \dot{g}^{\mathrm{t}}-\frac{\partial G}{\partial \xi} \dot{\xi}-\frac{\partial G}{\partial d} \dot{d}-\frac{\partial G}{\partial p} \dot{p}-\frac{\partial G}{\partial g^{\mathrm{tp}}} \dot{g}^{\mathrm{tp}} \\
& =\boldsymbol{\sigma}: \dot{\boldsymbol{\varepsilon}}^{\mathrm{t}}-\dot{g}^{\mathrm{t}}-\frac{\partial G}{\partial \xi} \dot{\xi}-\frac{\partial G}{\partial d} \dot{d}-g^{\mathrm{tp}}, \\
r & =-c_{0} \dot{\theta}-\theta \boldsymbol{\alpha}: \dot{\boldsymbol{\sigma}}+\gamma_{\mathrm{loc}},
\end{aligned}
$$

To proceed with the definition of the evolution equations associated with the various physical mechanisms, we consider that the evolution of all inelastic strains is completely related to the underlying process of phase transformation, as assumed for the TRIP effect elsewhere (Lagoudas and Entchev, 2004; Chemisky et al., 2014) and captured herein in (A.6). The following specific evolution equations are then considered, where all the rate quantities are linked with the rate of change of the martensite volume fraction:

$$
\begin{aligned}
\dot{\varepsilon}^{\mathrm{tt}-\mathrm{F}} & =\Lambda^{\mathrm{tt}-\mathrm{F}} \dot{\xi}^{\mathrm{F}}, \\
\dot{g}^{\mathrm{F}} & =f^{\mathrm{t}-\mathrm{F}} \dot{\xi}^{\mathrm{F}}, \\
\dot{p}^{\mathrm{F}} & =f^{\mathrm{tp}-\mathrm{F}} \dot{\xi}^{\mathrm{F}}, \\
\dot{\varepsilon}^{\mathrm{tp}-\mathrm{F}}=\Lambda^{\mathrm{tp}-\mathrm{F}} \dot{p}^{\mathrm{F}} & =\Lambda^{\mathrm{tp}-\mathrm{F}} f^{\mathrm{tp}-\mathrm{F}} \dot{\xi}^{\mathrm{F}}, \\
\dot{g}^{\mathrm{tp}-\mathrm{F}}=H^{\mathrm{tp}-\mathrm{F}} \dot{p}^{\mathrm{F}} & =f^{\mathrm{tp}-\mathrm{F}} \dot{\xi}^{\mathrm{F}}, \\
\dot{d}^{\mathrm{F}} & =f^{\mathrm{td}-\mathrm{F}} \dot{\xi}^{\mathrm{F}}, \\
\dot{\varepsilon}^{\mathrm{td}-\mathrm{F}}=\Lambda^{\mathrm{td}-\mathrm{F}} \dot{d} & =\Lambda^{\mathrm{td}-\mathrm{F}} f^{\mathrm{td}-\mathrm{F}} \dot{\xi}^{\mathrm{F}},
\end{aligned}
$$

and 


$$
\begin{aligned}
\dot{\varepsilon}^{\mathrm{tt}-\mathrm{R}} & =\Lambda^{\mathrm{tt}-\mathrm{R}} \dot{\xi}^{\mathrm{R}}, \\
\dot{g}^{\mathrm{R}} & =f^{\mathrm{t}-\mathrm{R}} \dot{\xi}^{\mathrm{R}}, \\
\dot{p}^{\mathrm{R}} & =f^{\mathrm{tp}-\mathrm{R}} \dot{\xi}^{\mathrm{R}}, \\
\dot{\varepsilon}^{\mathrm{tp}-\mathrm{R}}=\Lambda^{\mathrm{tp}-\mathrm{R}} \dot{p}^{\mathrm{R}} & =\Lambda^{\mathrm{tp}-\mathrm{R}} f^{\mathrm{tp}-\mathrm{R}} \dot{\xi}^{\mathrm{R}}, \\
\dot{g}^{\mathrm{tp}-\mathrm{R}}=H^{\mathrm{tp}-\mathrm{R}} \dot{p}^{\mathrm{R}} & =f^{t p-R} f^{\mathrm{tp}-\mathrm{R}} \dot{\xi}^{\mathrm{R}}, \\
\dot{d}^{\mathrm{R}} & =f^{\mathrm{td}-\mathrm{R}} \dot{\xi}^{\mathrm{R}}, \\
\dot{\varepsilon}^{\mathrm{td}-\mathrm{R}}=\Lambda^{\mathrm{td}-\mathrm{R}} \dot{d} & =\Lambda^{\mathrm{td}-\mathrm{R}} f^{\mathrm{td}-\mathrm{R}} \dot{\xi}^{\mathrm{R}} .
\end{aligned}
$$

blueIn the above equations, $\Lambda^{\text {tt-F }}$ represents the evolution tensor (that is, the direction of the strain rate) for the recoverable part of the transformation strain during forward transformation, while $\Lambda^{\mathrm{tp}-\mathrm{F}}$ and $\boldsymbol{\Lambda}^{\mathrm{td}-\mathrm{F}}$ represent the irrecoverable part related to plasticity and damage, respectively (during forward transformation). During reverse transformation, the three evolution tensors are denoted as $\Lambda^{\mathrm{tt}-\mathrm{R}}, \boldsymbol{\Lambda}^{\mathrm{tp}-\mathrm{R}}$ and $\boldsymbol{\Lambda}^{\mathrm{td}-\mathrm{R}}$. The functional forms $f^{\mathrm{tp}-\mathrm{F}}$ and $f^{\mathrm{td}-\mathrm{F}}$ relate the magnitude of the hardening energy with the rate of change of the martensite during forward transformation, and with the amount of damage with the rate of change of the martensite during forward transformation, respectively. During reverse transformation, those quantities are denoted as $f^{\mathrm{tp}-\mathrm{R}}$ and $f^{\mathrm{td}-\mathrm{R}}$. the energetic conjugates to the internal variables denoted $\boldsymbol{A}=-\frac{\partial G}{\partial \boldsymbol{\zeta}}$ (cf. (A.15)), it is deducted that the generalized thermodynamic forces related to transformation and the associated evolutions of transformation strain, transformation hardening energy, accumulated transformation-induced plastic strain, and damage are given as: 
- Forward transformation ( $\left.\operatorname{set} \boldsymbol{A}_{\mathrm{F}}\right)$ :

$$
\begin{aligned}
& A_{\xi^{\mathrm{F}}}=A_{\xi}=\frac{1}{2} \boldsymbol{\sigma}: \Delta \mathcal{S}: \boldsymbol{\sigma}+\boldsymbol{\sigma}: \Delta \boldsymbol{\alpha}\left(\theta-\theta_{0}\right)-\rho \Delta c\left[\left(\theta-\theta_{0}\right)-\theta \ln \left(\frac{\theta}{\theta_{0}}\right)\right]+\rho \Delta s_{0} \theta-\rho \Delta E_{0}, \\
& \boldsymbol{A}_{\boldsymbol{\varepsilon}^{\mathrm{F}}}=A_{\boldsymbol{\varepsilon}^{\mathrm{tt}-\mathrm{F}}}=A_{\boldsymbol{\varepsilon}^{\mathrm{tp}-\mathrm{F}}}=A_{\boldsymbol{\varepsilon}^{\mathrm{td}-\mathrm{F}}}=A_{\boldsymbol{\varepsilon}^{\mathrm{t}}}=\boldsymbol{\sigma}, \\
& A_{g^{\mathrm{F}}}=-1 \\
& A_{p^{\mathrm{F}}}=A_{p}=0 \\
& A_{g^{\mathrm{tp}-\mathrm{F}}}=-1 \\
& A_{d^{\mathrm{F}}}=A_{d}=\frac{1}{2(1-d)^{2}} \boldsymbol{\sigma}: \mathcal{S}: \boldsymbol{\sigma},
\end{aligned}
$$

- Reverse transformation (set $\left.\boldsymbol{A}_{\mathrm{R}}\right)$ :

$$
\begin{aligned}
& A_{\xi^{\mathrm{R}}}=-A_{\xi}=-\frac{1}{2} \boldsymbol{\sigma}: \Delta \mathcal{S}: \boldsymbol{\sigma}-\boldsymbol{\sigma}: \Delta \boldsymbol{\alpha}\left(\theta-\theta_{0}\right)+\rho \Delta c\left[\left(\theta-\theta_{0}\right)-\theta \ln \left(\frac{\theta}{\theta_{0}}\right)\right]-\rho \Delta s_{0} \theta+\rho \Delta E_{0}, \\
& \boldsymbol{A}_{\boldsymbol{\varepsilon}^{\mathrm{R}}}=A_{\boldsymbol{\varepsilon}^{\mathrm{tt}-\mathrm{R}}}=A_{\boldsymbol{\varepsilon}^{\mathrm{tp}-\mathrm{R}}}=A_{\boldsymbol{\varepsilon}^{\mathrm{td}-\mathrm{R}}}=A_{\boldsymbol{\varepsilon}^{\mathrm{t}}}=\boldsymbol{\sigma}, \\
& A_{g^{\mathrm{t}-\mathrm{R}}}=1 \\
& A_{p^{\mathrm{R}}}=A_{p}=0 \\
& A_{g^{\mathrm{tp}-\mathrm{R}}}=-1 \\
& A_{d^{\mathrm{R}}}=A_{d}=\frac{1}{2(1-d)^{2}} \boldsymbol{\sigma}: \mathcal{S}: \boldsymbol{\sigma} .
\end{aligned}
$$

\subsection{Transformation limits}

Since phase transformation, TRIP, and damage are assumed to be rate-independent phenomena, a threshold for the activation of such mechanisms that depends primarily on thermodynamic forces should be defined, (Edelen, 1974). Specifically, the evolution of all internal variables $\dot{\zeta}$ should respect the following, where $\mathcal{S}$ is a domain in the space of the components of $\boldsymbol{A}$ having boundary $\partial \mathcal{S}$ :

$$
\begin{aligned}
& \dot{\boldsymbol{\zeta}}=0 \quad \rightarrow \boldsymbol{A} \in \mathcal{S}+\partial \mathcal{S} \\
& \dot{\boldsymbol{\zeta}} \neq 0 \quad \rightarrow \boldsymbol{A} \in \partial \mathcal{S}
\end{aligned}
$$

Following the methodology introduced by Germain (1973) and referred as generalized standard materials by Halphen and Nguyen (1975), if $\partial \mathcal{S}$ is a surface with a continuous 
tangent plane and if $\Phi(\boldsymbol{A})$ is a function continuously differentiable with respect to $\boldsymbol{A}$, zero on $\partial \mathcal{S}$, and negative in $\mathcal{S}$, then one can write:

$$
\begin{aligned}
& \boldsymbol{A} \in \mathcal{S}, \quad \dot{\boldsymbol{\zeta}}=0 \\
& \boldsymbol{A} \in \partial \mathcal{S}, \quad \dot{\boldsymbol{\zeta}}=\dot{\lambda} \operatorname{grad} \Phi \quad \Leftrightarrow \quad \dot{\boldsymbol{\zeta}}_{\alpha}=\dot{\lambda} \frac{\partial \Phi}{\partial \boldsymbol{A}_{\alpha}}, \dot{\lambda} \geq 0 .
\end{aligned}
$$

Further, if state variables are included as parameters and the domain $\mathcal{S}$ remains convex, the second law of thermodynamics is satisfied and maximum dissipation principle as well (Halphen and Nguyen, 1975).

Note that the processes of forward and reverse transformations are considered independently, in the sense that dissipation related to the rate of the internal variables defined for forward transformation $\boldsymbol{\zeta}^{\mathrm{F}}$ and $\boldsymbol{\zeta}^{\mathrm{R}}$ (cf. (16) and (17)) should be independently non-negative, i.e.:

$$
\boldsymbol{A}_{\mathrm{F}}: \dot{\boldsymbol{\zeta}}^{\mathrm{F}} \geq 0 ; \boldsymbol{A}_{\mathrm{R}}: \dot{\boldsymbol{\zeta}}^{\mathrm{F}} \geq 0
$$

The two criteria for forward and reverse transformations are based on the ones proposed elsewhere (Qidwai and Lagoudas (2000); Lagoudas et al. (2012); Chatziathanasiou et al. $(2015))$ :

$$
\begin{aligned}
& \Phi^{\mathrm{F}}=\hat{\Phi}^{\mathrm{F}}+A_{\xi^{\mathrm{F}}}-f^{\mathrm{t}-\mathrm{F}}(\xi)+H^{\mathrm{tp}} p-Y^{\mathrm{t}-\mathrm{F}} \\
& \Phi^{\mathrm{R}}=-\hat{\Phi}^{\mathrm{R}}+A_{\xi^{\mathrm{R}}}+f^{\mathrm{t}-\mathrm{R}}(\xi)-H^{\mathrm{tp}} p+Y^{\mathrm{t}-\mathrm{R}}
\end{aligned}
$$

Those two functions are null on the surfaces $\partial \mathcal{S}_{\mathrm{F}}$ and $\partial \mathcal{S}_{\mathrm{R}}$ of the convex domains $\mathcal{S}_{\mathrm{F}}$ and $\mathcal{S}_{\mathrm{R}}$, respectively, if the important functions $\hat{\Phi}^{\mathrm{F}}(\boldsymbol{\sigma})$ and $\hat{\Phi}^{\mathrm{R}}(\boldsymbol{\sigma})$ are convex.

Considering (19) and assuming that $\dot{\lambda}$ is a positive multiplier, the rate of the internal variables are given as: 


$$
\begin{aligned}
& \dot{\xi}^{\mathrm{F}}=\dot{\lambda} \frac{\partial \Phi^{\mathrm{F}}}{\partial A_{\xi^{\mathrm{F}}}}=\dot{\lambda}, \quad \dot{\boldsymbol{\varepsilon}}^{\mathrm{tt}-\mathrm{F}}=\dot{\lambda} \frac{\partial \Phi^{\mathrm{F}}}{\partial \boldsymbol{A}_{\varepsilon^{\mathrm{tt}-\mathrm{F}}}}=\dot{\xi}^{\mathrm{F}} \frac{\partial \Phi^{\mathrm{F}}}{\partial \boldsymbol{\sigma}} \\
& \dot{\xi}^{\mathrm{R}}=\dot{\lambda} \frac{\partial \Phi^{\mathrm{R}}}{\partial A_{\xi^{\mathrm{R}}}}=\dot{\lambda}, \quad \dot{\boldsymbol{\varepsilon}}^{\mathrm{tt}-\mathrm{R}}=\dot{\lambda} \frac{\partial \Phi^{\mathrm{R}}}{\partial \boldsymbol{A}_{\varepsilon^{\mathrm{tt}-\mathrm{R}}}}=\dot{\xi}^{\mathrm{R}} \frac{\partial \Phi^{\mathrm{R}}}{\partial \boldsymbol{\sigma}} .
\end{aligned}
$$

Comparing (14) and (15) we see:

$$
\begin{aligned}
\boldsymbol{\Lambda}^{\mathrm{tt}-\mathrm{F}} & =\frac{\partial \Phi^{\mathrm{F}}}{\partial \boldsymbol{\sigma}}, \\
\boldsymbol{\Lambda}^{\mathrm{tt}-\mathrm{R}} & =\frac{\partial \Phi^{\mathrm{R}}}{\partial \boldsymbol{\sigma}} .
\end{aligned}
$$

\subsection{Choice of Functional Forms}

\subsubsection{Fully recoverable martensitic transformation}

The transformation functions $\hat{\Phi}^{F}$ and $\hat{\Phi}^{R}$ are the particular terms in the transformation criteria that consider the shape of the bounding surfaces in the six-dimensional stress hyperspace; here a modified Prager function is chosen that accounts for tension-compression asymmetry but not anisotropy (Bouvet et al., 2004; Grolleau et al., 2011). The following formulation closely follows Patoor et al. (1996), Peultier et al. (2008), and Chemisky et al. (2011). It predicts that the initiation of SMA forward transformation depends on the stress tensor invariants and asymmetry-related parameters. Specifically,

$$
\hat{\Phi}^{\mathrm{F}}(\boldsymbol{\sigma})=\left(\sqrt{3 J_{2}(\boldsymbol{\sigma})}\left[1+b \frac{J_{3}(\boldsymbol{\sigma})}{J_{2}^{3 / 2}(\boldsymbol{\sigma})}\right]^{\frac{1}{n}}-k_{\sigma}\right) H^{c u r}(\boldsymbol{\sigma}) .
$$

The terms $J_{2}(\boldsymbol{\sigma})$ and $J_{3}(\boldsymbol{\sigma})$ denote the second and third invariants of the deviatoric part $\boldsymbol{\sigma}^{\prime}$. These are given as:

$$
J_{2}(\boldsymbol{\sigma})=\frac{1}{2} \sigma_{i j}^{\prime} \sigma_{i j}^{\prime}, \quad \text { and } \quad J_{3}(\boldsymbol{\sigma})=\frac{1}{3} \sigma_{i j}^{\prime} \sigma_{j k}^{\prime} \sigma_{k i}^{\prime}
$$

using summation notation for repeated indices. Constants $b$ and $n$ are associated with the ratio between stress magnitudes needed to induce forward transformation under tension and compression loading. Convexity is ensured under specific conditions detailed in Chatziathanasiou et al. (2015). 
The evolution of the maximum transformation strain $H^{c u r}$ is represented by the following decaying exponential function (Hartl et al., 2010a):

$$
H^{\operatorname{cur}}(\bar{\sigma})= \begin{cases}H_{\min } & ; \quad \bar{\sigma} \leq \bar{\sigma}_{\text {crit }}, \\ H_{\text {min }}+\left(H_{\text {sat }}-H_{\text {min }}\right)\left(1-e^{-k\left(\bar{\sigma}-\bar{\sigma}_{\text {crit }}\right)}\right) & ; \quad \bar{\sigma}>\bar{\sigma}_{\text {crit }}\end{cases}
$$

Here $\bar{\sigma}$ denotes the Mises stress and $H_{\min }$ corresponds to the minimal observable transformation strain magnitude generated during full transformation under tensile loading (or the two way shape memory strain magnitude). The parameter $H_{\text {sat }}$ describes the maximum possible recoverable full transformation strain generated under uniaxial tensile loading. Additionally, $\bar{\sigma}_{\text {crit }}$ denotes the critical Mises equivalent stress below which $H^{\text {cur }}=H_{\text {min }}$ and the parameter $k$ controls the rate at which $H^{\text {cur }}$ exponentially evolves from $H_{\min }$ to $H_{\text {sat }}$.

The threshold for forward transformation introduced in (3.2) is not constant and is given as (Lagoudas et al., 2012):

$$
Y^{\mathrm{t}-\mathrm{F}}=Y^{\mathrm{crit}-\mathrm{F}}+D \boldsymbol{\sigma}: \Lambda_{\varepsilon} \mathrm{F}
$$

The variables $D$ and $Y^{\text {crit-F }}$ are model constants associated with the differing influences of stress on transformation temperatures for forward and reverse transformation. They are calculated from knowledge of other material constants (Lagoudas et al., 2012).

During forward transformation, the transformation strain is oriented in the direction of the applied stress, which motivates the selected $J_{2}-J_{3}$ form of the direction tensor $\Lambda^{\mathrm{t}-\mathrm{F}}$. During reverse transformation, it is assumed that the direction of transformation strain recovery is instead governed by the average orientation of the martensite. This is represented in an average sense by the value of the macroscopic transformation strain $\varepsilon^{\mathrm{tt}}=\varepsilon^{\mathrm{tt}-\mathrm{F}}+\varepsilon^{\mathrm{tt}-\mathrm{R}}$ as normalized by the martensite volume fraction $\xi$. Specifically, we assume

$$
\Lambda^{\mathrm{t}-\mathrm{R}}=\frac{\varepsilon^{\mathrm{tt}-\mathrm{F}}}{\xi^{F}}+\frac{\varepsilon^{\mathrm{tt}-\mathrm{R}}}{\xi^{R}} .
$$

Given the assumed associativity for the reverse transformation strain (see (23)), the transformation function $\hat{\Phi}^{\mathrm{R}}$ for reverse transformation is then expressed as:

$$
\hat{\Phi}^{\mathrm{R}}=\boldsymbol{\sigma}\left(\frac{\varepsilon^{\mathrm{tt}-\mathrm{F}}}{\xi^{F}}+\frac{\varepsilon^{\mathrm{tt}-\mathrm{R}}}{\xi^{R}}\right) .
$$


After (27), the threshold for reverse transformation is expressed as:

$$
Y^{\mathrm{t}-\mathrm{R}}=Y^{\mathrm{crit}-\mathrm{R}}-D \boldsymbol{\sigma}: \bar{\varepsilon}^{\mathrm{t}}
$$

where $Y^{\text {crit-R }}$ is another material constant (usually taken equal to $Y^{\text {crit-F }}$ ).

An evolution equation also links the time rate of changes of the hardening energies $\left(\dot{g}^{\mathrm{F}}\right.$ and $\dot{g}^{\mathrm{R}}$ ) with those of martensite $\left(\dot{\xi}^{\mathrm{F}}\right.$ and $\left.\dot{\xi}^{\mathrm{R}}\right)$, according to (14) and (15). Then $f^{\mathrm{t}-\mathrm{F}}$ and $f^{\mathrm{t}-\mathrm{R}}$ are referred to as the forward and reverse hardening functions, respectively, which define the current transformation hardening behavior. Note that $g^{\mathrm{t}}$, being a contribution to the Gibbs free energy, cannot depend on the time derivative of the martensitic volume fraction but only on the transformation history. As per (14) and (15), the evolution equation associated with $g^{\mathrm{t}}$ changes with the transformation direction such that, given the reversibility of martensitic transformation in SMAs, in the absence of other dissipative mechanisms the Gibbs free energy should take on the same value for the same state of the external variables upon completion of any full transformation loop. If all contributions to the Gibbs free energy with the exception of $g^{\mathrm{t}}$ are returned to their original values after a full transformation loop, the following condition must be satisfied to fully return $G^{r}$ to its initial state:

$$
\int_{0}^{1} f^{\mathrm{t}-\mathrm{F}} d \xi+\int_{1}^{0} f^{\mathrm{t}-\mathrm{R}} d \xi=0 .
$$

This necessary condition restricts the choice of hardening function for forward and reverse transformations and constrains the calibration accordingly. The specification of a form for the hardening functions that describe smooth transition from elastic to transformation response is another key contribution of the model proposed by Lagoudas et al. (2012):

$$
\begin{aligned}
f^{\mathrm{t}-\mathrm{F}}(\xi) & =\frac{1}{2} a_{1}\left(1+\xi^{n_{1}}-(1-\xi)^{n_{2}}\right)+a_{3}, \\
f^{\mathrm{t}-\mathrm{R}}(\xi) & =\frac{1}{2} a_{2}\left(1+\xi^{n_{3}}-(1-\xi)^{n_{4}}\right)-a_{3} .
\end{aligned}
$$

Here, $n_{1}, n_{2}, n_{3}$ and $n_{4}$ are real exponents in the interval $(0,1]^{1}$. This form is selected here

\footnotetext{
${ }^{1}$ If all four exponents equal 1, the original model of Boyd and Lagoudas (1996) is recovered, see Appendix A of Lagoudas et al. (2012).
} 
since it is specifically adapted to the response of polycrystalline SMA systems wherein the transformation hardening can be quite "smooth", especially after the completion of several cycles. Such smoothness is tuned by the adjustment of the parameters $\left\{n_{1}, n_{2}, n_{3}, n_{4}\right\}$.

\subsubsection{Forms related to the evolution equations associated with damage}

The damage accumulation functions $f^{\mathrm{td}-\mathrm{F}}\left(\hat{\Phi}^{\mathrm{fwd}}\right)$ and $f^{\mathrm{td}-\mathrm{R}}\left(\hat{\Phi}^{\mathrm{rev}}\right)$ are based on a linear accumulation law (Lemaitre and Chaboche, 2002) written in terms of the integer number $\mathrm{N}$ of cycles completed such that,

$$
\frac{\Delta d}{D_{\text {crit }}}=\frac{\Delta N}{N_{f}}
$$

where $N_{f}$ and $D_{\text {crit }}$ are the number of cycles and local damage associated with local catastrophic failure, respectively. Note that failure will be defined as the state at which $d$ reaches the critical value $D_{\text {crit }}$.

This linear accumulation law can be written to consider continuous evolutions over time:

$$
\frac{\mathrm{d} d}{D_{\text {crit }}}=\frac{\mathrm{d} N}{N_{f}} \Rightarrow, \frac{\dot{d}}{D_{\text {crit }}}=\frac{\dot{N}}{N_{f}} .
$$

Considering fatigue occurs only as a consequence of transformation cycles (full or partial) and that a full cycle corresponds in the evolution of the martensitic volume fraction from 0 to 1 and back to $0,(34)$ can be rewritten to consider both forward and reverse transformations as $(\operatorname{see}(14),(15))$

$$
\begin{gathered}
\dot{d}^{\mathrm{F}}=\dot{\xi}^{\mathrm{F}} \frac{D_{\text {crit }}}{2 N_{f}}=\dot{\xi}^{\mathrm{F}} f^{\mathrm{td}-\mathrm{F}} \\
\dot{d}^{\mathrm{R}}=\dot{\xi}^{\mathrm{R}} \frac{D_{\text {crit }}}{2 N_{f}}=\dot{\xi}^{\mathrm{R}} f^{\mathrm{td}-\mathrm{R}} .
\end{gathered}
$$

In this way, the damage accumulation functions are defined. blueWhile it is postulated in Section 3 that damage may evolve actively during forward transformation, here we propose a general formulation that considers damage evolution during both forward and reverse transformation. Future experimental studies will be needed to ascertain the relative importance of forward versus reverse transformation as mechanisms for damage evolution.

From previous experimental studies, it has been shown that the fatigue life $N_{f}$ of SMA actuators is correlated to the cyclic mechanical work they perform (Calhoun et al., 2015). 
blueDuring isobaric uniaxial fatigue testing (the main response motivating this more general study), the actuation work per unit of volume done in each half cycle by a constant uniaxial stress $\sigma$ distributed homogeneously over a specimen generating uniaxial strain $\varepsilon^{\mathrm{t}}$ is the product $\sigma \varepsilon^{\mathrm{t}}$. As an empirical measure, this so-called actuation work neglects the small inelastic permanent strains generated during a single transformation cycle such that $\sigma \varepsilon^{\mathrm{t}} \simeq$ $\sigma \varepsilon^{\mathrm{tt}-\mathrm{f}}$. It was shown that a power law was sufficient to capture cycles to failure per

$$
N_{f}=\left(\frac{\sigma \varepsilon^{\mathrm{tt}-\mathrm{f}}}{C^{d}}\right)^{-\gamma_{d}}
$$

Examining (24) in such a case of uniaxial loading and assuming small values of $b$, we see $\left.\hat{\Phi}^{\mathrm{F}}\right|_{\text {uniax }}=\sigma H^{\text {cur }}(\sigma)$. For the full transformation considered in these motiviating studies, $H^{\text {cur }}(\sigma)=\varepsilon^{\mathrm{tt}-\mathrm{f}}$ by definition of $(26)$, and thus $\left.\hat{\Phi}^{\mathrm{F}}\right|_{\text {uniax }}=\sigma \varepsilon^{\mathrm{tt}-\mathrm{f}}$. Motivated by this relationship in this work, we then make a generalized equivalence between the effectiveness of the past power law and its applicability in three dimensions (pending future multi-axial studies). Finally, noting that in the case of full transformation under proportional loading (e.g., in the uniaxial case), it can be shown that $\hat{\Phi}^{\mathrm{F}}=\hat{\Phi}^{\mathrm{R}}$. This allows us to then introduce

$$
N_{f}=\left(\frac{\hat{\Phi}^{\mathrm{F}}}{C^{d}}\right)^{-\gamma_{d}}-N_{f}^{0}=\left(\frac{\hat{\Phi}^{\mathrm{R}}}{C^{d}}\right)^{-\gamma_{d}}-N_{f}^{0} .
$$

Here, $N_{f}^{0}$ is a parameter linked to the actuation work required for a static failure $\left(N_{f}=0\right)$, while $C^{d}$ and $\gamma_{d}$ are parameters characteristic of the number of cycles to failure dependence on actuation work. Combining (35) and (37) and currently assuming that damage accumulates equally during forward and reverse transformation, the final form of the damage functions are:

$$
\begin{aligned}
& f^{\mathrm{td}-\mathrm{F}}(\hat{\Phi})=\frac{D_{\text {crit }}}{2}\left[\left(\frac{\hat{\Phi}^{\mathrm{F}}}{C^{d}}\right)^{-\gamma_{d}}-N_{f}^{0}\right]^{-1}, \\
& f^{\mathrm{td}-\mathrm{R}}(\hat{\Phi})=\frac{D_{\text {crit }}}{2}\left[\left(\frac{\hat{\Phi}^{\mathrm{R}}}{C^{d}}\right)^{-\gamma_{d}}-N_{f}^{0}\right]^{-1} .
\end{aligned}
$$

Such forms, obtained from observations of isobaric uniaxial experiments, substantially defines the evolution of damage and is applicable for a wide range of thermomechanical loadings. Obviously, a large experimental effort is required to validate this critical extension from 
one-dimensional (uniaxial) to three-dimensional, where such conditions as non-proportional loading or partial transformation must be considered; in this work only isobaric actuation cycles will be considered in the discussions of experimental validation.

\subsubsection{Forms of the evolution equations associated with plasticity}

The transformation plasticity magnitude function $f^{t p}(\xi)$ is inspired by past works (Lagoudas and Entchev, 2004; Chemisky et al., 2014). Several conclusions are drawn considering also the experimental observations by Wheeler et al. (2015) from actuation fatigue tests where specimens were thermally cycled under various constant stress levels (see Fig.3): ${ }^{2}$

Figure 3: Transformation and plastic strain evolution in NiTiHf actuators under various isobaric loads (Wheeler et al., 2015) (a-c) and the comparison of these three (d). The strains are measured at high and low temperature, with a zero reference based on the beginning of the first cooling cycle. No necking is observed in any sample.

- For moderate stress levels (200MPa; Fig. 3a), after a rapid increase in accumulated plastic strain, a stable regime is observed (after 1000 cycles). The plastic strain accumulates linearly from cycle to cycle during this stable regime up to the point of failure. Similar response has been observed on Ni60Ti40 alloys (Agboola et al., 2012) and NiTi alloys (Lagoudas, 2008).

- For higher actuation stress levels (400 MPa; Fig. 3b), a transient regime is first observed, as with the moderate stress levels. While an apparent stable regime is observed, one can observe a slight increase in plastic strain accumulation from cycle to cycle prior to failure.

- At the highest feasible stress levels (600 MPa; Fig. 3c), the same initial transient regime is observed; after which an apparent stabilized regime is also observed, followed by an important and continuous increase of the plastic strain rate up to failure.

\footnotetext{
${ }^{2}$ It is important to note that all tests considered were not associated with any observed localized necking behavior and that nearly constant applied stress can be assumed.
} 
blueFrom these experiments the effect of stress amplitude is clear. At high stress levels the rate of change of the irrecoverable strain increases from about the half lifetime of the sample. This behavior is characteristic of a change in the material's response, and is generally explained through stress concentration due to the development of defects (Van Humbeeck, 1991; Hornbogen, 2004). The functional form of the irrecoverable strain evolution should therefore account for that effect by considering a coupling with damage above a critical stress threshold, since this coupling is only observed at a high stress. The following evolution law for plastic strains is thus proposed:

$$
\begin{aligned}
& f^{\mathrm{tp}-\mathrm{F}}(p)=w^{\mathrm{tp}} C_{0}^{\mathrm{tp}}\left[\left(\frac{\hat{\Phi}^{\mathrm{F}}}{C_{\mathrm{tp}}}\right)^{\gamma_{\mathrm{tp}}}\left(C_{1}^{\mathrm{tp}} p+e^{\frac{-p}{C_{2}^{\mathrm{tp}}}}\right)+\left(\frac{\left\langle\bar{\sigma}-\sigma_{\mathrm{tp}}^{\mathrm{Y}}\right\rangle}{\sigma_{\mathrm{tp}}^{\mathrm{Y}}}\right)^{\alpha_{\mathrm{tp}}} \lambda_{\mathrm{tp}}\right] \\
& f^{\mathrm{tp}-\mathrm{R}}(p)=\left(1-w^{\mathrm{tp}}\right) C_{0}^{\mathrm{tp}}\left[\left(\frac{\hat{\Phi}^{\mathrm{R}}}{C_{\mathrm{tp}}}\right)^{\gamma_{\mathrm{tp}}}\left(C_{1}^{\mathrm{tp}} p+e^{\frac{-p}{C^{\mathrm{tp}}}}\right)+\left(\frac{\left\langle\bar{\sigma}-\sigma_{\mathrm{tp}}^{\mathrm{Y}}\right\rangle}{\sigma_{\mathrm{tp}}^{\mathrm{Y}}}\right)^{\alpha_{\mathrm{tp}}} \lambda_{\mathrm{tp}}\right],
\end{aligned}
$$

with

$$
\begin{aligned}
& \lambda_{\text {tp }}=\lambda_{\text {tp }}\left(\frac{d}{D_{\text {crit }}}, 1-\frac{D_{\text {coa }}}{D_{\text {crit }}}, p_{0}\right),=\hat{\lambda}_{\text {tp }}\left(\hat{d}, \hat{D}, p_{0}\right) \\
& \hat{\lambda}_{\mathrm{tp}}\left(\hat{d}, \hat{D}, p_{0}\right)=\left\{\begin{array}{l}
p_{0} \hat{d}(1-\hat{d})^{-2} \quad \hat{d}<=h \\
p_{0}\left((1-h)^{-2}+2 h(1-h)^{-3}(\hat{d}-h)+h(1-h)^{-2}\right) \quad \hat{d}>h,
\end{array}\right.
\end{aligned}
$$

with $h=1-\hat{D}$. The function $\lambda_{\text {tp }}$ is a typical level set power law function that depends on the current value of damage, the critical value for damage $D_{\text {crit }}$, and a constant $D_{\text {coa }}$ that indicates the change of regime of the evolution of plastic strains.

\section{4. blueMethodology of Model Parameters Identification}

The entire three-dimensional constitutive model for shape memory alloys experiencing cycling fatigue requires four sets of parameters to be calibrated:

- The thermoelastic model parameters,

- The parameters associated with phase transformation criteria (e.g., the conventional phase diagram), 
- The parameters characteristic of damage accumulation,

- The parameters characteristic of TRIP accumulation.

These are summarized in Table 1. The thermoelastic parameters of martensite and austenite blue(e.g., Young's moduli, coefficients of thermal expansion, Poisson's ratios) are usually calibrated from mechanical and thermal uniaxial loadings, where loads are applied at temperatures outside of transformation regions. The parameters qualifying the phase diagram $\left(M_{s}, M_{f}, A_{s}, A_{f}, C^{A}, C^{M}\right)$ along with those contained in the functional form of the maximum transformation strain $H^{\text {cur }}$ are calibrated based on several isobaric thermal cycles prior to the accumulation of substantial damage or TRIP.

The identification of the thermodynamical parameters of the model $\left(\rho \Delta \eta_{0}, \rho \Delta E_{0}, a_{1}, a_{2}\right.$, $\left.a_{3}, Y_{0}^{t}, D\right)$ and the material parameters for phase transformation are detailed in Lagoudas et al. (2012). blueAccording to the complexity of the functional forms, especially for the evolution of damage and TRIP, the parameters are generally identified utilizing an optimization algorithm that minimizes a cost function, defined as a square difference between experimental measurement and the simulated response, following the methodology found in Meraghni et al. (2011). The algorithm utilized in this work is a combined gradient based - genetic optimization scheme, which is used to successfully determine the transformation characteristic of three-dimensional SMA structures Chemisky et al. (2015). blueThe suggested identification procedure, used to present the validation cases in the next section, consists of the following sequence:

1. blueDetermination of the parameters for transformation strain $H^{\text {cur }}$ functional form via the optimization algorithm (objective function defined in terms of transformation strain magnitude with respect to a stress value).

2. blueDetermination of the other phase transformation parameters with reconstruction of the phase diagram from isobaric thermal cycles.

3. Determination of the fatigue damage parameters used the optimization algorithm to predict the number of cycles to failure according to the actuation energy. Using uniaxial isobaric loading, this last quantity is obtained experimentally for various stress levels. The parameters $C_{d}, \gamma^{d}, N_{f}^{0}$ can be evaluated with this procedure, ensuring that these 
parameters are positive values. The parameter $D_{\text {crit }}$ has been estimated at 0.1 from the experimental observation of crack density in the observed fatigue samples just prior to failure.

4. blueThe parameters for the evolution of TRIP are evaluated using the evolution of the uniaxial irrecoverable strain $\varepsilon^{p}$ with respect to the number of cycles. In the present approach, the parameter $D_{\text {coa }}$ has been set up to 0.05 (50\% of $\left.D_{\text {crit }}\right)$, since it is clear that a change of regime occurs the mid-life of the NiTiHf actuators loaded high stress (see Fig.3c), attributed to the evolution of damage. Note that $0 \leq D_{\text {coa }} \leq D_{\text {crit }} \leq 1$. The parameter identification of $w^{\text {tp }}$ requires some specific thermomechanical loading path and usually takes values greater than 0.5 and is constrained to stay within the range $0 \leq w^{\text {tp }} \leq 1$ (Chemisky et al., 2014). Since the half-cycles were not available in the database utilized to identify the parameters of the Ni60Ti40 and NiTiHf alloys, this value has been arbitrarily set to 0.6 .

5. blueThe remaining parameters $C_{0}^{\mathrm{tp}}, C_{1}^{\mathrm{tp}}, C^{\mathrm{tp}}, \gamma_{\mathrm{tp}}, C_{2}^{\mathrm{tp}}$ $\sigma_{\text {tp }}^{\mathrm{Y}}, \alpha^{\mathrm{tp}}, p_{0}$ have been identified utilizing the optimization algorithm based on the experimental results of the evolution of irrecoverable plastic strain as a function of the number of cycles. All these parameters must be positive values.

Table 1: Required material parameters and associated material properties

\begin{tabular}{c|c|c}
\hline Parameter Type & Set of Constants & Specific Response \\
\hline Thermoelastic properties & $\begin{array}{c}\text { Young's moduli, Poisson's ratios, } \\
\text { Coefficients of therm. expan., etc. }\end{array}$ & \\
\hline Phase transformation & $M_{s}, M_{f}, A_{s}, A_{f}, C^{A}, C^{M}$ & Phase diagram \\
& $H_{m i n}, H_{s a t}, k, \bar{\sigma}_{c r i t}$ & Transformation strain \\
\hline Damage & $n_{1}, n_{2}, n_{3}, n_{4}$ & Smoothness of transformation \\
\hline TRIP & $D_{\mathrm{crit}}, C_{d}, \gamma^{d}, N_{f}^{0}$ & Evolution law for damage \\
\hline & $w^{\mathrm{tp}}, C_{0}^{\mathrm{tp}}, C_{1}^{\mathrm{tp}}, C^{\mathrm{tp}}, \gamma_{\mathrm{tp}}, C_{2}^{\mathrm{tp}}$ & First and second stage \\
\hline
\end{tabular}




\section{Comparison of Experimental Results}

This new model for the description of functional and structural damage has been specifically formulated to capture the combined effect of phase transformation, transformationinduced plasticity and fatigue damage of polycrystalline SMAs subjected to general threedimensional thermomechanical loading and has been implemented in the 'smartplus' library (Chemisky, 2016). While the capabilities of such a modeling approach to capture the effects of phase transformation have been already demonstrated by Lagoudas et al. (2012) and Chatziathanasiou et al. (2016), here we specifically consider the evolution of damage and TRIP strains.

The set of experiments utilized to validate the proposed model consider specimens loaded uniaxially to different constant stress levels(i.e., in the austenitic condition) and then subjected to thermally-induced transformation cycles up to failure. The parameters are taken from Wheeler et al. (2015), where NiTiHf actuators were tested at three relatively high constant stress levels (i.e., 200, 400 and 600 MPa., cf.Fig. 3) blueacross a temperature range from approximately $300 \mathrm{~K}$ to $500 \mathrm{~K}$. A fourth stress level of $300 \mathrm{MPa}$ is used for validation since the full characterization of the elastic response was not addressed in the source work, standard values for NiTiHf alloys are applied. An average of transformation strain magnitudes generated over full cycles at each stress level is used to define the average experimental value shown in Fig. 4 and Fig. 5.

Figure 4: Dependence of maximum transformation strain magnitude on applied stress for the considered NiTiHf alloy (Wheeler et al., 2015). Data is fit using the functional form $H^{\text {cur }}(26)$ based on the average recovered transformation strain over all cycles at each stress levels considered.

The parameters that define the evolution equation for the damage internal variable have been identified based on the number of cycles to failure of the SMA actuators thermally cycled at different stress levels blue and are displayed in Table 2. The comparison between the fatigue database for various stress levels and the model simulation is presented in Figure 5, 
where the actuation energy density in this one-dimensional (uniaxial) setting is equivalent to blue $\hat{\Phi}=\hat{\Phi}^{\mathrm{F}}=\hat{\Phi}^{\mathrm{R}}=\sigma H^{\text {cur }}(\sigma)$.. Note that the stress levels of 200, 400 and $600 \mathrm{MPa}$ have been used for the calibration of the damage model, while data for the stress level of $300 \mathrm{MPa}$ (2 tests) are used to validate predictions.

The parameters related to the evolution of TRIP strains have been identified based on the evolution of residual strains as measured at high temperature (i.e., in the austenitic condition). The parameter identification algorithm used is a hybrid genetic - gradientbased method developed by Chemisky et al. (2015) and applied here to the least-square difference between the experimental and numerical irrecoverable strains for the three stress levels tested (i.e, 200, 400 and $600 \mathrm{MPa}$ ). It is noted, according to the comparison presented in Figure 7, that both functional fatigue (i.e., TRIP) and structural fatigue (i.e., total life) are accurately captured by the model, since both the number of cycles to failure and the level of irrecoverable strain are correctly described for the three stress levels tested. All three stages of plastic strain evolution with cycles are represented, and the rapid accumulation of TRIP strain magnitude towards the end of the lifetime of the actuator is clearly visible in the simulated results. The typical behavior of an actuator is represented here. ${ }^{3}$ Note in particular that the upward shift in transformation temperatures with increasing cycle count

\footnotetext{
${ }^{3}$ The transformation temperatures, not provided in Wheeler et al. (2015), are calibrated here using common values.
}

Figure 5: Number of cycles to failure as a function of the actuation energy $\hat{\Phi}$ for the considered NiTiHf alloy. Results from the isobaric tests performed at 200, 400, and $600 \mathrm{MPa}$ used for calibration; data from $300 \mathrm{MPa}$ tests used for validation.

Figure 6: blueSpectrum of evolution of damage with respect to the number of cycles. Number of failure from the isobaric tests performed at 200, 400, and $600 \mathrm{MPa}$ utilized for calibration in blue dot, $300 \mathrm{MPa}$ validation tests in orange. 
is captured.

Figure 7: Comparison between the evolution of irrecoverable strains in NiTiHf actuators under various isobaric loads (Wheeler et al., 2015) with the model simulations: a), b), and c) show comparisons of the evolution of TRIP strains for the calibration stress levels of 200, 400, and $600 \mathrm{MPa}$, respectively; d) shows an example of a simulation of the evolution of the response of an actuator for the first, $100^{t h}$, $200^{t h}$, and the last $\left(309^{t h}\right)$ cycle prior to failure. The blue and red dots correspond to the experimentally measured strains at high and low temperature for the considered cycles, respectively.

The second experiment (Fig. 8) used to validate the model focuses only on functional fatigue, whereby (i.e., TRIP) an SMA actuator is subjected to 80 thermal transformation cycles under constant load corresponding to a uniaxial stress level of $200 \mathrm{MPa}$ (Lagoudas, 2008), bluewhere the derived properties are given in Table 3. The actuator is at an early stage of its expected lifetime, so only the first and second stage of the evolution of plastic strain are captured, see Fig. 8a). Note that the evolution of the transformation strain with temperature is well captured, and that the shift of the transformation temperatures between the $1^{\text {st }}$ and the $80^{\text {th }}$ cycle is again accurately described by the proposed model (see Fig. $8 \mathrm{~b}$ ).

Figure 8: Comparison between the evolution of irrecoverable strains of a NiTi actuator under an isobaric load (uniaxial stress level of $200 \mathrm{MPa}$; Lagoudas (2008)) with the model simulations: a) comparisons of the evolution of TRIP strains; b) comparison of the full strain-temperature response of an actuator for the first and $80^{\text {th }}$ cycles.

blueAdditional numerical simulations have been performed for multiaxial actuation based on the same actuator to demonstrate the effect of the stress components on the actuation response. In a first case the SMA actuator is subjected to 80 thermal transformation cycles under constant load corresponding to a biaxial stress level, with $\left(\sigma_{11}=50, \sigma_{12}=\sigma_{21}=100\right.$, all other components of the stress tensor being 0 ). In the second case the biaxial stress level has be set to $\left(\sigma_{11}=100, \sigma_{12}=\sigma_{21}=50\right.$, all other components of the stress tensor being 0) The evolution of the non-zero total strain components with respect to temperature is 
shown in Fig. 9. While the evolution equation for irrecoverable strain is based on the Mises equivalent stress, it is seen that the irrecoverable strain as well as transformation strain follows the direction of the imposed stress. Also, the importance of shear stress component versus uniaxial stress component is highlighted here, regarding the amount of irrecoverable strain..

Figure 9: Comparison between the evolution of irrecoverable strains of a NiTi actuator of an actuator for the first and $80^{\text {th }}$ cycles, considering two multiaxial isobaric loads : 1) continuous grey line : $\sigma_{11}=50 \mathrm{MPa}$, $\sigma_{12}=\sigma_{21}=100 \mathrm{MPa}$, all other components of the stress tensor being 0$)$ and 2$)$ dashed grey line $\left(\sigma_{11}=\right.$ $100 \mathrm{MPa}, \sigma_{12}=\sigma_{21}=50 \mathrm{MPa}$, all other components of the stress tensor being 0 ). The evolution of a) comparison of the total uniaxial strain $\left(\varepsilon_{11}\right)$-temperature response, b) comparison of the total shear strain $\left(\varepsilon_{12}\right)$-temperature response. 
Table 2: Identified model parameters for the NiTiHf alloy

\begin{tabular}{|c|c|}
\hline Model Parameters & Identified value \\
\hline blue $E^{A}=E^{M}$ & $70000 \mathrm{MPa}$ \\
\hline blue $\nu^{A}=\nu^{M}$ & 0.3 \\
\hline blue $\alpha^{A}=\alpha^{M}$ & $0 K^{-1}$ \\
\hline blue $M_{s}, M_{f}, A_{s}, A_{f}$ & $293 \mathrm{~K}, 273 \mathrm{~K}, 313 \mathrm{~K}, 333 \mathrm{~K}$ \\
\hline blue $C^{A}=C^{M}$ & $7 \mathrm{MPa} . \mathrm{K}^{-1}$ \\
\hline blue $_{1}=n_{2}=n_{3}=n_{4}$ & 0.2 \\
\hline$H_{\min }$ & 0.005 \\
\hline$H_{\text {sat }}$ & 0.0277 \\
\hline$k$ & $0.0172 \mathrm{MPa}^{-1}$ \\
\hline $\bar{\sigma}_{c r i t}$ & $120 \mathrm{MPa}$ \\
\hline$b$ & 0 \\
\hline$n$ & 2 \\
\hline$D_{\text {crit }}$ & 0.14 \\
\hline & 0.07 \\
\hline & 85689.2 MPa \\
\hline & 1.040 \\
\hline$N_{f}^{0}$ & 7000 cycles \\
\hline$w^{\text {tp }}$ & 0.6 \\
\hline$C_{0}^{\mathrm{tp}}$ & 0.000245 \\
\hline$C_{1}^{\mathrm{tp}}$ & 0.000667 \\
\hline$C^{\text {tp }}$ & $6.144682 \mathrm{MPa}$ \\
\hline$\gamma_{\mathrm{tp}}$ & 4.132985 \\
\hline$C_{2}^{\mathrm{tp}}$ & 0.006239 \\
\hline$\sigma_{\mathrm{tp}}^{\mathrm{Y}}$ & $300 \mathrm{MPa}$ \\
\hline$\alpha^{\mathrm{tp}}$ & 3.720168 \\
\hline$p_{0}$ & 1.861436 \\
\hline
\end{tabular}


Table 3: Identified model parameters for the NiTi alloy

\begin{tabular}{|c|c|}
\hline Model Parameters & Identified value \\
\hline blue $E^{A}, E^{M}$ & $47000 \mathrm{MPa}, 24000 \mathrm{MPa}$ \\
\hline blue $\nu^{A}=\nu^{M}$ & 0.3 \\
\hline blue $\alpha^{A}=\alpha^{M}$ & $0 K^{-1}$ \\
\hline blue $M_{s}, M_{f}, A_{s}, A_{f}$ & $277.15 \mathrm{~K}, 260.15 \mathrm{~K}, 275.15 \mathrm{~K}, 291.15 \mathrm{~K}$ \\
\hline blue $C^{A}, C^{M}$ & $8.3,6.7 \mathrm{MPa} . \mathrm{K}^{-1}$ \\
\hline blue $n_{1}=n_{2}=n_{3}=n_{4}$ & 0.1 \\
\hline$H_{\min }$ & 0.05 \\
\hline$H_{\text {sat }}$ & 0.05 \\
\hline$k$ & $\mathrm{~N} / \mathrm{A}$ \\
\hline $\bar{\sigma}_{\text {crit }}$ & $\mathrm{N} / \mathrm{A}$ \\
\hline$b$ & 0 \\
\hline$n$ & 2 \\
\hline$w^{\text {tp }}$ & 0.6 \\
\hline$C_{0}^{\mathrm{tp}}$ & 0.019994 \\
\hline$C_{1}^{\mathrm{tp}}$ & 0.086058 \\
\hline$C^{\mathrm{tp}}$ & $22.564369 \mathrm{MPa}$ \\
\hline$\gamma_{\mathrm{tp}}$ & 3.322290 \\
\hline$C_{2}^{\mathrm{tp}}$ & 0.015947 \\
\hline$\sigma_{\mathrm{tp}}^{\mathrm{Y}}, \alpha^{\mathrm{tp}}, p_{0}$ & $\mathrm{~N} / \mathrm{A}$ \\
\hline
\end{tabular}




\section{Conclusions}

To simulate functional and structural fatigue in shape memory alloy actuators, a new phenomenological model has been proposed that considers the coupled accumulation of damage and transformation-induced plasticity and is inspired by recent three-dimensional models for phase transformation based on thermodynamics of irreversible processes. Structural fatigue is described using an evolution equation for damage based on the rate of transformation energy relative to the martensite volume fraction $\hat{\Phi}$. Such a description succeeds in capturing the number of cycles to failure of the SMA actuators thermally cycled at different stress levels. The evolution of irrecoverable strains (i.e., functional fatigue) is described based on the same rate of transformation energy, especially to describe the first (transient) and second (steady-state) stages of transformation-induced plastic strain evolution. To represent the third stage (accelerated accumulation), a power law that depends on the level of accumulated damage is applied to represent the effect of structural fatigue on the development of irrecoverable strains. It is demonstrated that this formulation can accurately describe the accumulation of TRIP strains for the three considered actuators loaded at different stress levels. It is finally shown that the expression of the transformation limits represent the shift in transformation temperatures observed during cycling loading of actuators. These various aspects combine to make this model the most complete description of shape memory alloy fatigue to date.

\section{References}

B. O. Agboola, D. J. Hartl, and D. C. Lagoudas. A Study of Actuation Fatigue of Shape Memory Alloy. In Volume 2: Mechanics and Behavior of Active Materials; Integrated System Design and Implementation; Bio-Inspired Materials and Systems; Energy Harvesting, page 287. ASME, sep 2012.

F. Auricchio, A. Constantinescu, C. Menna, and G. Scalet. A shakedown analysis of high cycle fatigue of shape memory alloys. International Journal of Fatigue, 87:112-123, jun 2016. 
T. Bartel and K. Hackl. A micromechanical model for martensitic phase-transformations in shape-memory alloys based on energy-relaxation. ZAMM, 89(10):792-809, oct 2009.

T. Bartel, A. Menzel, and B. Svendsen. Thermodynamic and relaxation-based modeling of the interaction between martensitic phase transformations and plasticity. Journal of the Mechanics and Physics of Solids, 59(5):1004-1019, 2011.

T. Baxevanis and D. C. Lagoudas. Fracture mechanics of shape memory alloys: review and perspectives. International Journal of Fracture, 191(1-2):191-213, feb 2015.

O. W. Bertacchini, D. C. Lagoudas, and E. Patoor. Thermomechanical transformation fatigue of TiNiCu SMA actuators under a corrosive environment - Part I: Experimental results. International Journal of Fatigue, 31(10):1571-1578, 2009.

B. Bhattacharya and B. Ellingwood. Continuum damage mechanics analysis of fatigue crack initiation. International Journal of Fatigue, 20(9):631-639, oct 1998.

V. Birman. Review of Mechanics of Shape Memory Alloy Structures. Applied Mechanics Reviews, 50(11):629, 1997.

Z. Bo and D. C. Lagoudas. Thermomechanical Modeling of Polycrystalline $\{$ SMA $\}$ s Under Cyclic Loading, \{Part $\mathrm{I}\}:\{$ Theoretical $\}$ D $\}$ erivations. International Journal of Engineering Science, 37:1089-1140, 1999a.

Z. Bo and D. C. Lagoudas. Thermomechanical modeling of polycrystalline SMAs under cyclic loading, Part III: Evolution of plastic strains and two-way shape memory effect. International Journal of Engineering Science, 37(9):1175-1203, jul 1999b.

N. Bonora, D. Gentile, A. Pirondi, and G. Newaz. Ductile damage evolution under triaxial state of stress: Theory and experiments. International Journal of Plasticity, 21(5):981$1007,2005$.

C. Bouvet, S. Calloch, and C. Lexcellent. A phenomenological model for pseudoelasticity of shape memory alloys under multiaxial proportional and nonproportional loadings. European Journal of Mechanics, A/Solids, 23(1):37-61, 2004. 
J. Boyd and D. Lagoudas. A thermodynamical constitutive model for shape memory materials. Part I. The monolithic shape memory alloy. International Journal of Plasticity, 12 (6):805-842, 1996.

M. Brünig. An anisotropic ductile damage model based on irreversible thermodynamics. International Journal of Plasticity, 19(10):1679-1713, 2003.

C. Calhoun, R. Wheeler, T. Baxevanis, and D. C. Lagoudas. Actuation fatigue life prediction of shape memory alloys under the constant-stress loading condition. Scripta Materialia, 95(1):58-61, 2015.

D. Chatziathanasiou, Y. Chemisky, F. Meraghni, G. Chatzigeorgiou, and E. Patoor. Phase Transformation of Anisotropic Shape Memory Alloys: Theory and Validation in Superelasticity. Shape Memory and Superelasticity, 1(3):359-374, aug 2015.

D. Chatziathanasiou, Y. Chemisky, G. Chatzigeorgiou, and F. Meraghni. Modeling of coupled phase transformation and reorientation in shape memory alloys under nonproportional thermomechanical loading. International Journal of Plasticity, 82:192-224, 2016.

G. Chatzigeorgiou, N. Charalambakis, Y. Chemisky, and F. Meraghni. Periodic homogenization for fully coupled thermomechanical modeling of dissipative generalized standard materials. International Journal of Plasticity, 81, 2016.

G. Chatzigeorgiou, N. N. Charalambakis, Y. Chemisky, and F. Meraghni. Thermomechanical behavior of dissipative composite materials. ISTE Press - Elsevier, 2018.

Y. Chemisky. smartplus: Smart Material Algoritjms and Research Tools, 2016.

Y. Chemisky, A. Duval, E. Patoor, and T. Ben Zineb. Constitutive model for shape memory alloys including phase transformation, martensitic reorientation and twins accommodation. Mechanics of Materials, 43(7):361-376, 2011.

Y. Chemisky, G. Chatzigeorgiou, P. Kumar, and D. C. Lagoudas. A constitutive model for cyclic actuation of high-temperature shape memory alloys. Mechanics of Materials, 68: 120-136, 2014. 
Y. Chemisky, F. Meraghni, N. Bourgeois, S. Cornell, R. Echchorfi, and E. Patoor. Analysis of the deformation paths and thermomechanical parameter identification of a shape memory alloy using digital image correlation over heterogeneous tests. International Journal of Mechanical Sciences, 96-97:13-24, 2015.

C. Cisse, W. Zaki, and T. Zineb. A review of constitutive models and modeling techniques for shape memory alloys. International Journal of Plasticity, 76:244-284, aug 2015.

B. D. Coleman and M. E. Gurtin. Thermodynamics with Internal State Variables. The Journal of Chemical Physics, 47(2):597-613, 1967.

V. Dattoma, S. Giancane, R. Nobile, and F. W. Panella. Fatigue life prediction under variable loading based on a new non-linear continuum damage mechanics model. International Journal of Fatigue, 28(2):89-95, 2006.

F. Desrumaux, F. Meraghni, and M. L. Benzeggagh. Generalised Mori-Tanaka Scheme to Model Anisotropic Damage Using Numerical Eshelby Tensor. Journal of Composite Materials, 35(07):603-624, apr 2001.

D. G. B. Edelen. On the Characterization of Fluxes in Nonlinear Irreversible Thermodynamics. International Journal of Engineering Science, 12:397-411, 1974.

G. Eggeler, E. Hornbogen, A. Yawny, A. Heckmann, and M. Wagner. Structural and functional fatigue of NiTi shape memory alloys. Materials Science and Engineering A, 378(1-2 SPEC. ISS.):24-33, 2004.

B. Fedelich. A stochastic theory for the problem of multiple surface crack coalescence. International Journal of Fracture, 91(1):23-45, 1998.

A. M. Figueiredo, P. Modenesi, and V. Buono. Low-cycle fatigue life of superelastic NiTi wires. International Journal of Fatigue, 31(4):751-758, 2009.

A. Fischer. A special newton-type optimization method. Optimization, 24(3-4):269-284, jan 1992. 
P. Germain. Cours de Mécanique des Miliex Continus: Tome I-Théorie Générale. Masson Et Cie, Paris, 1973.

P. Germain, Q. S. Nguyen, and P. Suquet. Continuum thermodynamics. Journal of Applied Mechanics, 50:1010-1020, 1983.

J. Grasa, M. A. Perez, J. A. Bea, J. M. Garcia-Aznar, and M. Doblare. A probabilistic damage model for acrylic cements. Application to the life prediction of cemented hip implants. In International Journal of Fatigue, volume 27, pages 891-904, 2005.

V. Grolleau, H. Louche, V. Delobelle, A. Penin, G. Rio, Y. Liu, and D. Favier. Assessment of tension-compression asymmetry of NiTi using circular bulge testing of thin plates. Scripta Materialia, 65(4):347-350, aug 2011.

X. Gu, Z. Moumni, W. Zaki, and W. Zhang. Shakedown based model for high-cycle fatigue of shape memory alloys. Smart Materials and Structures, 25(11):115012, nov 2016.

B. Halphen and Q. S. Nguyen. Sur les matériaux standards généralisés. Journal de Mécanique, 14(1):39-63, 1975.

D. Hartl, D. Lagoudas, J. Mabe, F. Calkins, and J. Mooney. Use of $\{\mathrm{N}\} \mathrm{i} 60\{\mathrm{~T}\}$ i Shape Memory Alloy for Active Jet Engine Chevron Application, $\{\mathrm{P}\}$ art $\{\mathrm{II}\}:\{\mathrm{E}\}$ xperimentally Validated Numerical Analysis. Smart Materials and Structures, 19(1), 2010a.

D. J. Hartl and D. C. Lagoudas. Aerospace applications of shape memory alloys. Proceedings of the Institution of Mechanical Engineers, Part G: Journal of Aerospace Engineering, 221 (4):535-552, jan 2007.

D. J. Hartl and D. C. Lagoudas. Constitutive modeling and structural analysis considering simultaneous phase transformation and plastic yield in shape memory alloys. Smart Materials and Structures, 18:1-17, 2009.

D. J. Hartl, G. Chatzigeorgiou, and D. C. Lagoudas. Three-dimensional modeling and numerical analysis of rate-dependent irrecoverable deformation in shape memory alloys. International Journal of Plasticity, 26(10):1485-1507, 2010b. 
F. Hild, C. Denoual, P. Forquin, and X. Brajer. On the probabilistic-deterministic transition involved in a fragmentation process of brittle materials. Computers and Structures, 81 (12):1241-1253, 2003.

R. Hojjati-Talemi and M. A. Wahab. Fretting fatigue crack initiation lifetime predictor tool: Using damage mechanics approach. Tribology International, 60:176-186, 2013.

E. Hornbogen. Review Thermo-mechanical fatigue of shape memory alloys, 2004.

Q. Kan, G. Kang, W. Yan, Y. Dong, and C. Yu. An energy-based fatigue failure model for super-elastic NiTi alloys under pure mechanical cyclic loading. page 84090F. International Society for Optics and Photonics, apr 2012.

G. Kang, Q. Kan, C. Yu, D. Song, and Y. Liu. Whole-life transformation ratchetting and fatigue of super-elastic NiTi Alloy under uniaxial stress-controlled cyclic loading. Materials Science and Engineering: A, 535:228-234, feb 2012.

H. E. Karaca, E. Acar, H. Tobe, and S. M. Saghaian. NiTiHf-based shape memory alloys. Materials Science and Technology, 30(13):1530-1544, nov 2014.

W. Khalil, A. Mikolajczak, C. Bouby, and T. Ben Zineb. A constitutive model for Fe-based shape memory alloy considering martensitic transformation and plastic sliding coupling: Application to a finite element structural analysis. Journal of Intelligent Material Systems and Structures, 23:1143-1160, 2012.

A. Khandelwal and V. Buravalla. Models for Shape Memory Alloy Behavior: An overview of modeling approaches. The International Journal of Structural Changes in Solids, 1(1): $111-148,2009$.

B. Kiefer, T. Bartel, and A. Menzel. Implementation of numerical integration schemes for the simulation of magnetic SMA constitutive response. Smart Materials and Structures, 21(9):094007, sep 2012.

P. Ladeveze, E. LeDantec, P. Ladevèze, and E. Le Dantec. Damage modelling of the elementary ply for laminated composites. Composites Science and Technology, 43(3):257-267, 1992. 
D. Lagoudas, D. Hartl, Y. Chemisky, L. Machado, and P. Popov. Constitutive model for the numerical analysis of phase transformation in polycrystalline shape memory alloys. International Journal of Plasticity, 32-33:155-183, 2012.

D. C. Lagoudas. Shape memory alloys: modeling and engineering applications. Springer, New York, USA, 2008.

D. C. Lagoudas, P. B. Entchev, P. Popov, E. Patoor, L. C. Brinson, and X. Gao. Shape Memory Alloys - Part $\{\mathrm{II}\}$ : Modeling of polycrystals. Mechanics of Materials, 38(5-6): 430-462, 2006.

D. C. Lagoudas, G. Chatzigeorgiou, and D. J. Kumar. Modeling and Experimental Study of Simultaneous Creep and Transformation in Polycrystalline High-Temperature Shape Memory Alloys. Journal of Intelligent Material Systems and Structures, 20(18):2257-2267, 2009a.

D. C. Lagoudas, D. A. Miller, L. Rong, and P. K. Kumar. Thermomechanical fatigue of shape memory alloys. Smart Materials and Structures, 18(8):085021, 2009b.

D. C. Lagoudas and P. B. Entchev. Modelling of transformation-induced plasticity and its effect on the behavior of porous shape memory alloys. Part I: Constitutive model for fully dense $\{$ SMA $\}$ s. Mechanics of Materials, 36(9):865-892, 2004.

D. C. Lagoudas, C. Li, D. A. Miller, L. Rong, and C. Li. Thermomechanical Transformation Fatigue of $\{\mathrm{SMA}\}$ Actuators. In Proceedings of SPIE, Smart Structures and Materials, volume 3992, pages 420-429, 2000.

J. Lemaitre and J. L. Chaboche. Mechanics of solid materials. Cambridge University Press, Cambridge, UK, 2002.

J. Lemaitre and R. Desmorat. Engineering damage mechanics: Ductile, creep, fatigue and brittle failures. 2005.

J. Lemaitre, R. Desmorat, and M. Sauzay. Anisotropic damage law of evolution. European Journal of Mechanics - A/Solids, 19(2):187-208, mar 2000. 
B. T. Lester, T. Baxevanis, Y. Chemisky, and D. C. Lagoudas. Review and perspectives: shape memory alloy composite systems, 2015.

J. Lubliner. On the thermodynamic foundations of non-linear solid mechanics. International Journal of Non-Linear Mechanics, 7(3):237-254, 1972.

M. Luo, M. Dunand, and D. Mohr. Experiments and modeling of anisotropic aluminum extrusions under multi-axial loading Part II: Ductile fracture. International Journal of Plasticity, 32:36-58, 2012.

Z. Mahboob, Y. Chemisky, F. Meraghni, and H. Bougherara. Mesoscale modelling of tensile response and damage evolution in natural fibre reinforced laminates. Composites Part B: Engineering, 119:168-183, 2017.

C. Maletta, E. Sgambitterra, F. Furgiuele, R. Casati, and A. Tuissi. Fatigue of pseudoelastic NiTi within the stress-induced transformation regime: a modified CoffinManson approach. Smart Materials and Structures, 21(11):112001, nov 2012.

C. Maletta, E. Sgambitterra, F. Furgiuele, R. Casati, and A. Tuissi. Fatigue properties of a pseudoelastic NiTi alloy: Strain ratcheting and hysteresis under cyclic tensile loading. International Journal of Fatigue, 66:78-85, sep 2014.

G. A. Maugin. The thermomechanics of plasticity and fracture. Cambridge University Press, Cambridge, UK, 1992.

F. Meraghni, F. Desrumaux, and M. L. Benzeggagh. Implementation of a constitutive micromechanical model for damage analysis in glass mat reinforced composite structures. Composites Science and Technology, 62(16):2087-2097, 2002.

F. Meraghni, H. Nouri, N. Bourgeois, C. Czarnota, and P. Lory. Parameters identification of fatigue damage model for short glass fiber reinforced polyamide (PA6-GF30) using digital image correlation. Procedia Engineering, 10:2110-2116, 2011.

J. Mohd Jani, M. Leary, A. Subic, and M. A. Gibson. A review of shape memory alloy research, applications and opportunities. Materials \{ \&\} Design, 56:1078-1113, apr 2014. 
Z. Moumni, A. Van Herpen, and P. Riberty. Fatigue analysis of shape memory alloys: energy approach. Smart Materials and Structures, 14(5):S287-S292, 2005.

S. Nemat-Nasser and W. G. Guo. Superelastic and cyclic response of NiTi SMA at various strain rates and temperatures. Mechanics of Materials, 38(5-6):463-474, 2006.

A. Paiva, M. A. Savi, A. Paivia, and M. A. Savi. An Overview of Constitutive Models for Shape Memory Alloys. Mathematical Problems in Engineering, 2006:1-30, 2006.

E. Patoor, A. Eberhardt, and M. Berveiller. Micromechanical Modelling of Superelasticity in Shape Memory Alloys. Journal de Physique IV, 6(C1):277-292, 1996.

E. Patoor, D. C. Lagoudas, P. B. Entchev, L. C. Brinson, and X. Gao. Shape Memory Alloys, $\{\mathrm{P}\}$ art $\{\mathrm{I}\}$ : $\{\mathrm{G}\}$ eneral Properties and Modeling of Single Crystals. Mechanics of Materials, 38(5-6):391-429, 2006.

B. Peultier, T. Ben Zineb, and E. Patoor. A simplified micromechanical constitutive law adapted to the design of shape memory applications by finite element methods. Material Science \& Engineering A, 481-482:384-388, 2008.

M. A. Qidwai and D. C. Lagoudas. On the Thermodynamics and Transformation Surfaces of Polycrystalline $\{\mathrm{N}\} \mathrm{i}\{\mathrm{T}\}$ i Shape Memory Alloy Material. International Journal of Plasticity, 16:1309-1343, 2000.

P. Rosakis, A. J. Rosakis, G. Ravichandran, and J. Hodowany. A thermodynamic internal variable model for the partition of plastic work into heat and stored energy in metals. Journal of the Mechanics and Physics of Solids, 48:581-607, 2000.

C. C. Roth and D. Mohr. Effect of strain rate on ductile fracture initiation in advanced high strength steel sheets: Experiments and modeling. International Journal of Plasticity, 56: $19-44,2014$.

J. Rupil, L. Vincent, F. Hild, and S. Roux. Identification and Probabilistic Modeling of Mesocrack Initiations in 304L Stainless Steel. International Journal for Multiscale Computational Engineering, 9(4):445-458, 2011. 
L. Saint-Sulpice, S. Arbab Chirani, S. Calloch, S. A. Chirani, S. Calloch, S. Arbab Chirani, and S. Calloch. A 3D super-elastic model for shape memory alloys taking into account progressive strain under cyclic loadings. MOM, 41(1):12-26, 2009.

M. Schmidt-Baldassari. Numerical concepts for rate-independent single crystal plasticity. Computer Methods in Applied Mechanics and Engineering, 192(11):1261-1280, 2003.

G. Scirè Mammano and E. Dragoni. Functional fatigue of Ni-Ti shape memory wires under various loading conditions. International Journal of Fatigue, 69:71-83, 2014.

D. Shi, C. Dong, X. Yang, Y. Sun, J. Wang, and J. Liu. Creep and fatigue lifetime analysis of directionally solidified superalloy and its brazed joints based on continuum damage mechanics at elevated temperature. Materials and Design, 45:643-652, 2013.

J. C. Simo and J. W. Ju. Strain- and stress-based continuum damage models-I. Formulation. International Journal of Solids and Structures, 23(7):821-840, 1987.

J. Simo and T. Hughes. Computational Inelasticity, volume 7 of Interdisciplinary Applied Mathematics. Springer-Verlag, New York, 1998.

D. Song, G. Kang, Q. Kan, C. Yu, and C. Zhang. Damage-based life prediction model for uniaxial low-cycle stress fatigue of super-elastic NiTi shape memory alloy microtubes. Smart Materials and Structures, 24(8):085007, aug 2015.

D. Song, G. Kang, C. Yu, Q. Kan, and C. Zhang. Non-proportional multiaxial fatigue of super-elastic NiTi shape memory alloy micro-tubes: Damage evolution law and lifeprediction model. International Journal of Mechanical Sciences, 131-132:325-333, oct 2017.

H. Tobushi, T. Nakahara, Y. Shimeno, and T. Hashimoto. Low-Cycle Fatigue of TiNi Shape Memory Alloy and Formulation of Fatigue Life. Journal of Engineering Materials and Technology, 122(2):186, 2000.

J. Van Humbeeck. Cycling effects. Fatigue and degradation of shape memory alloys. Journal de physique IV, 1:C4-199, 1991. 
J. Van Humbeeck. Non-medical applications of shape memory alloys. Materials Science and Engineering: A, 273-275:134-148, 1999.

G. Z. Voyiadjis and P. I. Kattan. Advances in damage mechanics : metals and metal matrix composites. Elsevier, 1999.

G. Z. Voyiadjis and B. Deliktas. A coupled anisotropic damage model for the inelastic response of composite materials. Computer Methods in Applied Mechanics and Engineering, 183(3-4):159-199, 2000.

R. Wheeler, D. Hartl, Y. Chemisky, and D. Lagoudas. Modeling of thermo-mechanical fatigue and damage in shape memory alloy axial actuators. In Proceedings of SPIE - The International Society for Optical Engineering, volume 9432, 2015.

W. Zaki, S. Zamfir, and Z. Moumni. An extension of the ZM model for shape memory alloys accounting for plastic deformation. Mechanics of Materials, 42(3):266-274, 2010.

T. Zhang, P. E. McHugh, and S. B. Leen. Finite element implementation of multiaxial continuum damage mechanics for plain and fretting fatigue. International Journal of Fatigue, 44:260-272, 2012.

Y. Zhang, Y. You, Z. Moumni, G. Anlas, J. Zhu, and W. Zhang. Experimental and theoretical investigation of the frequency effect on low cycle fatigue of shape memory alloys. International Journal of Plasticity, 90:1-30, mar 2017.

\section{Appendix A. Appendix A: Fundamentals of Thermodynamics of irreversible processes}

blueConsidering a small strain $\varepsilon$ at a considered material point, the strong form of the first law of thermodynamics can be expressed as

$$
\dot{E}=\boldsymbol{\sigma}: \dot{\varepsilon}-\operatorname{div} \boldsymbol{q}+\rho \mathcal{R}
$$

where $\boldsymbol{q}$ is the heat flux, $\mathcal{R}$ denotes the heat sources per unit mass, and $\boldsymbol{\sigma}$ is the Cauchy stress. Similarly, the second law of thermodynamics is written in the strong form as (Chatzigeorgiou 
et al., 2016):

$$
\theta \dot{\eta}-\frac{\mathbf{q}}{\theta} \cdot \nabla \theta+\operatorname{div} \boldsymbol{q}-\rho \mathcal{R} \geq 0
$$

where $\eta=\rho \varsigma$ is the entropy per unit volume. Combining equations (A.1) and (A.2) to eliminate extra heat sources yields

$$
\gamma=\boldsymbol{\sigma}: \dot{\varepsilon}+\theta \dot{\eta}-\dot{E}-\frac{\boldsymbol{q}}{\theta} \cdot \nabla \theta \geq 0
$$

where $\gamma$ is the internal entropy production per unit volume. We can also define $r$ as the difference between the rates of the mechanical work and the internal energy of $\mathcal{Q}$ as the thermal energy per unit volume provided by external sources, which gives

$$
\begin{aligned}
& r=\boldsymbol{\sigma}: \dot{\varepsilon}-\dot{E}, \\
& \mathcal{Q}=-\operatorname{div} \boldsymbol{q}+\rho \mathcal{R} .
\end{aligned}
$$

Further, the internal entropy production can be split into two contributions, where $\gamma_{\text {loc }}$ is the local entropy production (or intrinsic dissipation) and $\gamma_{\text {con }}$ is the entropy production due to heat conduction, giving

$$
\gamma_{\mathrm{con}}=-\frac{\boldsymbol{q}}{\theta} \cdot \nabla \theta
$$

The two laws of thermodynamics can then be simply expressed as

$$
\begin{aligned}
& \mathcal{Q}+r=0 \quad \text { and } \\
& \gamma=\gamma_{\text {loc }}+\gamma_{\text {con }} \geq 0
\end{aligned}
$$

Combining equations (A.3), (A.5), and (A.6), one can re-express the first principle of thermodynamics as:

$$
\boldsymbol{\sigma}: \dot{\varepsilon}+\theta \dot{\eta}=\gamma_{\mathrm{loc}}+\dot{E}
$$

When designing a constitutive law, especially with the aim of tracking fatigue damage and permanent deformation in a material, it is very useful to separate the various mechanisms into categories (e.g., elastic or inelastic, reversible or irreversible, dissipative or non-dissipative) following the methodology proposed by Chatzigeorgiou et al. (2018). Some of these mechanisms are responsible for permanent changes in material microstructure. To describe all 
observable phenomena it is required to express $E$ in terms of the proper variables capable of expressing the material state under every possible thermomechanical loading path. Following the approach of Germain et al. (1983), the internal energy $E$ is taken to be a convex function with regards to its arguments: the strain tensor $\varepsilon$, the entropy $\eta$ and a set of internal state variables $\zeta$ such that

$$
E:=E(\varepsilon, \eta, \zeta)
$$

The following definitions for the derivatives of the internal energy are postulated:

$$
\boldsymbol{\sigma}=\frac{\partial E}{\partial \varepsilon}, \quad \theta=\frac{\partial E}{\partial \eta}
$$

For the purposes of further development, it is convenient to introduce the Gibbs free energy potential $G$ by employing the following partial Legendre transformation (Maugin (1992)):

$$
G(\boldsymbol{\sigma}, \theta, \boldsymbol{\zeta}):=E-\theta \eta-\boldsymbol{\sigma}: \varepsilon
$$

Considering (A.8),(A.9), and the chain rule one can show that

$$
\mathrm{d} E=\frac{\partial E}{\partial \boldsymbol{\varepsilon}}: \mathrm{d} \boldsymbol{\varepsilon}+\frac{\partial E}{\partial \eta} \mathrm{d} \eta+\frac{\partial E}{\partial \boldsymbol{\zeta}}: \mathrm{d} \boldsymbol{\zeta}=\boldsymbol{\sigma}: \mathrm{d} \boldsymbol{\varepsilon}+\theta \mathrm{d} \eta+\frac{\partial E}{\partial \boldsymbol{\zeta}}: \mathrm{d} \boldsymbol{\zeta} .
$$

Further considering (A.10), we have:

$$
\begin{aligned}
\mathrm{d} G & =\frac{\partial G}{\partial \boldsymbol{\sigma}}: \mathrm{d} \boldsymbol{\sigma}+\frac{\partial G}{\partial \theta} \mathrm{d} \theta+\frac{\partial G}{\partial \boldsymbol{\zeta}}: \mathrm{d} \boldsymbol{\zeta}=\mathrm{d} E-\eta \mathrm{d} \theta-\theta \mathrm{d} \eta-\boldsymbol{\sigma}: \mathrm{d} \boldsymbol{\varepsilon}-\boldsymbol{\varepsilon}: \mathrm{d} \boldsymbol{\sigma} \\
& =\boldsymbol{\sigma}: \mathrm{d} \boldsymbol{\varepsilon}+\theta \mathrm{d} \eta+\frac{\partial E}{\partial \boldsymbol{\zeta}}: \mathrm{d} \boldsymbol{\zeta}-\eta \mathrm{d} \theta-\theta \mathrm{d} \eta-\boldsymbol{\sigma}: \mathrm{d} \boldsymbol{\varepsilon}-\boldsymbol{\varepsilon}: \mathrm{d} \boldsymbol{\sigma} \\
& =-\boldsymbol{\varepsilon}: \mathrm{d} \boldsymbol{\sigma}-\eta \mathrm{d} \theta+\frac{\partial E}{\partial \boldsymbol{\zeta}}: \mathrm{d} \boldsymbol{\zeta} .
\end{aligned}
$$

Thus, it can be deduced that ${ }^{4}$

$$
\boldsymbol{\varepsilon}=-\frac{\partial G}{\partial \boldsymbol{\sigma}}, \quad \eta=-\frac{\partial G}{\partial \theta}
$$

Returning to the expression of thermodynamic laws, combining equations (A.7) and (A.10) leads to

$$
\gamma_{\mathrm{loc}}=-\varepsilon: \dot{\boldsymbol{\sigma}}-\eta \dot{\theta}-\dot{G}
$$

\footnotetext{
${ }^{4}$ The same result can be obtained by utilizing the methodology of Coleman and Gurtin (1967) for thermodynamics with internal state variables; however, the issues raised by Lubliner (1972) that limit the case to the elastic response should also be considered.
} 
Expressing $\dot{G}$ in terms of its arguments and using (A.13), the last expression reduces to

$$
\gamma_{\mathrm{loc}}=-\frac{\partial G}{\partial \boldsymbol{\zeta}}: \dot{\boldsymbol{\zeta}}=-\boldsymbol{A}: \dot{\boldsymbol{\zeta}}
$$

Equation (A.15), in conjunction with (A.6), is used to identify proper evolution equations for the internal state variables. Usually, the mechanical and thermal dissipations are assumed to be decoupled and non-negative, i.e. $\gamma_{\text {loc }} \geq 0$ and $\gamma_{\text {con }} \geq 0$.

\section{Appendix A. Appendix B: Numerical Resolution and Computation of the Tan- gent Moduli}

blue Such a constitutive model is intended to be applied to the scope of Finite Element Analyses (FEA). In most FEA softwares, the variables are updated following a procedure that include three loops. A loading step is typically partitioned in time increments and is denoted by $\Delta x$. The increment during the global FEA solver is denoted $\partial x$. The increment during the Newton-Raphson scheme in the material constitutive law, which is described below, is denoted by the symbol $\delta x$. Such steps consist in finding the updated value of the stress tensor and of the internal variables of the model. In a backward Euler fully implicit numerical scheme, the value of a given quantity $x$ is updated from the previous time step $n$ to the current $n+1$ per

$$
x^{(n+1)}=x^{(n)}+\Delta x^{(n+1)} .
$$

Such an implicit relation is usually solved iteratively during the FEA calculations, and the current value is updated from iteration $m$ to iteration $m+1$ per

$$
x^{(n+1)(m+1)}=x^{(n+1)(m)}+\partial x^{(n+1)(m)} .
$$

blueThe return mapping algorithm is used in the constitutive law algorithm and consists of two parts: i) Initially, it is assumed that no evolution of the internal variables occurs, thus the material behaves linearly. This allows it to consider a thermoelastic prediction of all the fields. In such a prediction, the stress tensor and are estimated, while the internal variables are set to their initial value at the beginning of the time increment; (ii) The stress tensor 
and the internal variables are corrected such that the solution meets the requirements of the specified constitutive law (the forward transformation, reverse transformation, or both). During the return mapping algorithm, the total current strain and temperature are held constant such that:

$$
\delta \varepsilon^{(n+1)(m+1)(k)}=0, \quad \delta \theta^{(n+1)(m+1)(k)}=0,
$$

where $k$ denotes the increment number during the correction loop. The system of KuhnTucker set of inequalities can be summarized as:

$$
\begin{aligned}
& \Phi^{\mathrm{F}}=\hat{\Phi}^{\mathrm{F}}+A_{\xi^{\mathrm{F}}}-f^{\mathrm{t}-\mathrm{F}}(\xi)+H^{\mathrm{tp}} p-Y^{\mathrm{t}-\mathrm{F}} \leq 0, \quad \dot{\xi}^{\mathrm{F}} \geq 0, \quad \dot{\xi}^{\mathrm{F}} \Phi^{\mathrm{F}} 0 \\
& \Phi^{\mathrm{R}}=-\hat{\Phi}^{\mathrm{R}}+A_{\xi^{\mathrm{R}}}+f^{\mathrm{t}-\mathrm{R}}(\xi)-H^{\mathrm{tp}} p+Y^{\mathrm{t}-\mathrm{R}} \leq 0, \quad \dot{\xi}^{\mathrm{R}} \geq 0, \quad \dot{\xi}^{\mathrm{R}} \Phi^{\mathrm{R}} 0 .
\end{aligned}
$$

Note that the size of the system to solve might therefore depends on the activated mech$\operatorname{anism}(\mathrm{s})$. We utilized the Fischer-Burmeister Fischer (1992) complementary function to replace the Kuhn-Tucker set of inequalities that typically results from dissipative mechanisms into a set of equations. Such formulation results in a smooth complementary problem (Kiefer et al., 2012), which does not require the information of the number of active sets. This methodology has already been utilized by Schmidt-Baldassari (2003) in the context of rate-independent multi-surface plasticity, by Bartel and Hackl (2009); Bartel et al. (2011) for martensitic phase transformation modeling, and by Kiefer et al. (2012) for the simulation of the constitutive response of magnetic SMAs.

blueAt this point the methodology presented in Chatziathanasiou et al. (2016) is briefly summarized here. The Fischer-Burmeister technique transforms a set of Kuhn-Tucker inequality into an equivalent equation :

$$
\Phi^{m} \leq 0 \quad \dot{s}^{m} \geq 0 \quad \Phi^{m} \dot{s}^{m}=0 \rightarrow \sqrt{\left(\Phi^{m}\right)^{2}+\left(\dot{s}^{m}\right)^{2}}+\Phi^{m}-\dot{s}^{m}=0
$$

This equation has two sets of roots: either $\Phi^{m} \leq 0 ; \quad \dot{s}^{m}=0$, which means that the mechanism $m$ is not activated, or $\Phi^{m}=0 ; \quad \dot{s}^{m} \geq 0$ indicates that the mechanism $m$ is activated and a solution for $\dot{s}^{m}$ (and consequently for all internal variables $\dot{\boldsymbol{V}}^{m}$ ) is searched. Next, the elastic prediction - inelastic correction method is utilized to solve the unconstrained system of equations using a Newton-Raphson scheme. The inelastic transformation strain is 
recalled here:

$$
\varepsilon^{\mathrm{t}}=\varepsilon^{\mathrm{F}}+\varepsilon^{\mathrm{R}}
$$

During a time increment $n$, at the $m$-th iteration of the solver and the $k$-th iteration of the constitutive law algorithm, the transformation strain thus writes:

$$
\boldsymbol{\varepsilon}_{t}^{(n+1)(m+1)(k)}=\boldsymbol{\varepsilon}_{m}^{\mathrm{F}(n+1)(m+1)(k)}+\boldsymbol{\varepsilon}_{m}^{\mathrm{R}(n+1)(m+1)(k)}
$$

To avoid lengthy expressions in the sequel, the iteration numbers will be omitted. Any quantity $x$ denotes the $x^{(n+1)(m+1)(k)}$, the increment $\Delta x$ denotes the $\Delta x^{(n+1)(m+1)(k)}$, the increment $\delta x$ denotes the $\delta x^{(n+1)(m+1)(k)}$ and the increment $\partial x$ denotes the $\partial x^{(n+1)(m+1)}$. The convex cutting plane (CCP) Simo and Hughes (1998); Qidwai and Lagoudas (2000) is utilized to approximate the evolution of the inelastic strain as:

$$
\begin{aligned}
& \varepsilon^{t}=\varepsilon^{\mathrm{t}(n+1)(m+1)(k-1)} \\
& +\left(\Lambda^{\mathrm{tt}-\mathrm{F}(n+1)(m+1)(k-1)}+\Lambda^{\mathrm{tp}-\mathrm{F}(n+1)(m+1)(k-1)} f^{\mathrm{tp}-\mathrm{F}(n+1)(m+1)(k-1)}\right. \\
& \left.+\boldsymbol{\Lambda}^{\mathrm{td}-\mathrm{F}(n+1)(m+1)(k-1)} f^{\mathrm{d}-\mathrm{F}(n+1)(m+1)(k-1)}\right) \delta \xi^{\mathrm{F}(n+1)(m+1)(k)} \\
& +\left(\Lambda^{\mathrm{tt}-\mathrm{R}(n+1)(m+1)(k-1)}+\Lambda^{\mathrm{tp}-\mathrm{R}(n+1)(m+1)(k-1)} f^{\mathrm{tp}-\mathrm{R}(n+1)(m+1)(k-1)}\right. \\
& \left.+\boldsymbol{\Lambda}^{\mathrm{td}-\mathrm{R}(n+1)(m+1)(k-1)} f^{\mathrm{d}-\mathrm{R}(n+1)(m+1)(k-1)}\right) \delta \xi^{\mathrm{R}(n+1)(m+1)(k)} \\
& =\boldsymbol{\varepsilon}^{\mathrm{t}(n+1)(m+1)(k-1)}+\Lambda^{\mathrm{F}(n+1)(m+1)(k-1)} \delta \xi^{\mathrm{F}(n+1)(m+1)(k)}+\Lambda^{\mathrm{R}(n+1)(m+1)(k-1)} \delta \xi^{\mathrm{R}(n+1)\left(m+\mathrm{A}^{\prime}\right)\left(\xi^{2}\right)}
\end{aligned}
$$

blueThe comparison and the efficiency evaluation of the convex cutting plane and the closest point projection has been discussed in detail in Qidwai and Lagoudas (2000), and it has been shown that the convex cutting plane algorithm is more efficient in most cases, even if it may require more steps to converge when strong non-proportional loadings are considered. The total current strain and temperature are held constant in displacement driven FEA. Assuming an additive decomposition of strains and the previously induced constitutive relations between elastic strain and stress, and thermal strain and temperature provides:

$$
\delta \mathcal{M}: \boldsymbol{\sigma}+\mathcal{M}: \delta \boldsymbol{\sigma}+\delta \boldsymbol{\alpha} \theta+\delta \varepsilon^{t}=0
$$


Since the variations of elastic compliance tensor and the thermal expansion tensor are dependent on the volume fraction of forward or reverse martensitic transformation:

$$
\delta \mathcal{M}=\Delta \mathcal{M}\left(\delta \xi^{\mathrm{F}}-\delta \xi^{\mathrm{F}}\right)
$$

and

$$
\delta \boldsymbol{\alpha}=\Delta \boldsymbol{\alpha}\left(\delta \xi^{\mathrm{F}}-\delta \xi^{\mathrm{R}}\right)
$$

it is therefore possible to define a total stress-influence evolution tensor, such as:

$$
\begin{gathered}
\boldsymbol{\kappa}^{\mathrm{F}} \delta \xi^{\mathrm{F}}=\left[\boldsymbol{\Lambda}^{\mathrm{F}}+\Delta \boldsymbol{\mathcal { M }}: \boldsymbol{\sigma}+\Delta \boldsymbol{\alpha}\left(\theta-\theta_{0}\right)\right] \delta \xi^{\mathrm{F}} \\
\boldsymbol{\kappa}^{\mathrm{F}} \delta \xi^{\mathrm{R}}=\left[\boldsymbol{\Lambda}^{\mathrm{R}}-\Delta \boldsymbol{M}: \boldsymbol{\sigma}-\Delta \boldsymbol{\alpha}\left(\theta-\theta_{0}\right)\right] \delta \xi^{\mathrm{F}}
\end{gathered}
$$

blueThus, with the help of (A.8), (A.10) and (A.11), (A.9) is now written as:

$$
\delta \boldsymbol{\sigma}=-\mathcal{L}: \sum_{j}\left[\boldsymbol{\kappa}^{j} \delta s^{j}\right]
$$

Recall that the transformations (forward and reverse) depend on the stress and the internal variables through the definition of thermodynamical forces. Applying the chain rule to these criterion yields:

$$
\begin{aligned}
\delta \Phi^{\mathrm{F}} & =\left[-\frac{\partial \Phi^{\mathrm{F}}}{\partial \boldsymbol{\sigma}}: \boldsymbol{\kappa}^{\mathrm{F}}+\mathcal{K}^{\mathrm{FF}}\right] \delta \xi^{\mathrm{F}}+\left[-\frac{\partial \Phi^{\mathrm{F}}}{\partial \boldsymbol{\sigma}}: \boldsymbol{\kappa}^{\mathrm{R}}+\mathcal{K}^{\mathrm{FR}}\right] \delta \xi^{\mathrm{R}} \\
\delta \Phi^{\mathrm{R}} & =\left[-\frac{\partial \Phi^{\mathrm{R}}}{\partial \boldsymbol{\sigma}}: \boldsymbol{\kappa}^{\mathrm{F}}+\mathcal{K}^{\mathrm{RF}}\right] \delta \xi^{\mathrm{F}}+\left[-\frac{\partial \Phi^{\mathrm{R}}}{\partial \boldsymbol{\sigma}}: \boldsymbol{\kappa}^{\mathrm{R}}+\mathcal{K}^{\mathrm{RR}}\right] \delta \xi^{\mathrm{R}}
\end{aligned}
$$

with:

$$
\mathcal{K}^{l j} \delta s^{j}=\sum_{i}\left(\frac{\partial \Phi^{l}}{\partial \boldsymbol{\xi}^{j}} \boldsymbol{\Lambda}^{j}\right) \delta s^{j}
$$

blue The numerical resolution consists of solving the following system of complementary Fischer-Burmeister functions using a Newton-Raphson scheme:

$$
\boldsymbol{F}+\delta \boldsymbol{F}=\mathbf{0}
$$

with:

$$
\begin{aligned}
\delta F^{i} & =\frac{\partial F^{i}}{\partial \Phi^{i}} \frac{\partial \Phi^{i}}{\partial s^{j}} \delta s^{j} \\
\delta F^{i} & =\frac{\partial F^{i}}{\partial \Phi^{i}} \mathcal{B}_{i j} \delta s^{j} .
\end{aligned}
$$


In the above equations, $\mathcal{B}$ is a matrix containing the partial derivatives of $\boldsymbol{\Phi}$, such that $\mathcal{B}_{l j}=\sum_{j}\left[-\frac{\partial \Phi^{l}}{\partial \boldsymbol{\sigma}} \boldsymbol{\kappa}^{j}+\mathcal{K}^{l j}\right]$. The iterative loops stop when the convergence criteria on all the complementary functions has been fulfilled.

Appendix A.1. Determination of the thermomechanical quantities and tangent moduli

blueSince we require the computation of the solver iteration increments $\Delta \varepsilon^{(n+1)(m+1)}=$ $\Delta \boldsymbol{\varepsilon}^{(n+1)(m)}+\partial \boldsymbol{\varepsilon}^{(n+1)(m)}$ the mechanical tangent modulus $\boldsymbol{D}^{\varepsilon}$ is required:

$$
\text { ð }=D^{\varepsilon} \partial \varepsilon
$$

To compute such quantities, the criterion is utilized and uses (A.14):

$$
ð \Phi^{l}=\frac{\partial \Phi^{l}}{\partial \boldsymbol{\sigma}}: \mathcal{L}(ð \boldsymbol{\varepsilon}-\boldsymbol{\alpha} \partial \theta)-\sum_{j}\left[\boldsymbol{\kappa}^{j} \xi^{j}\right]+\frac{\partial \Phi^{l}}{\partial \theta} \partial \boldsymbol{\theta}+\sum_{j} \mathcal{K}^{l j} \partial \xi^{j} .
$$

Considering now only the subset of activated mechanisms, i.e. the ones that satisfy

$$
ð \Phi^{l}=0
$$

the following holds true (the superscript $l$ shall now refer to any activated mechanism, and only those):

$$
\begin{aligned}
& ð \Phi^{l}=\frac{\partial \Phi^{l}}{\partial \boldsymbol{\sigma}}:\left(\mathcal{L}(ð \boldsymbol{\varepsilon}-\boldsymbol{\alpha} \precsim \theta)-\sum_{j}\left[\boldsymbol{\kappa}^{j} \boldsymbol{\partial}^{j}\right]\right)+\frac{\partial \Phi^{l}}{\partial \theta} \mathrm{\partial} \boldsymbol{\theta}+\sum_{j} \mathcal{K}^{l j} \boldsymbol{\partial}^{j}=0 \\
& \sum_{j}\left[\frac{\partial \Phi^{l}}{\partial \boldsymbol{\sigma}} \boldsymbol{\kappa}^{j}-\mathcal{K}^{l j}\right] ð \xi^{j}=\frac{\partial \Phi^{l}}{\partial \boldsymbol{\sigma}}: \mathcal{L}: \partial \boldsymbol{\varepsilon}+\left(\frac{\partial \Phi^{l}}{\partial \theta}-\frac{\partial \Phi^{l}}{\partial \boldsymbol{\sigma}}: \mathcal{L}: \boldsymbol{\alpha}\right) \partial \theta .
\end{aligned}
$$

The set of non-linear equations that can be rearranged in a matrix-like format:

$$
\hat{B} \boldsymbol{\xi}=\mathcal{C}
$$

where $\boldsymbol{\xi}=\left[\xi^{\mathrm{F}}, \xi^{\mathrm{R}}\right]$. The components of the reduced sensitivity tensor $\hat{\boldsymbol{B}}$ that correspond to the active load mechanism variables, with respect to the strain and temperature, are:

$$
\hat{\boldsymbol{B}}^{l j}=\frac{\partial \Phi^{l}}{\partial \boldsymbol{\sigma}}: \boldsymbol{\kappa}^{j}-\mathcal{K}^{l j}
$$


and

$$
\mathcal{C}^{l}=\frac{\partial \Phi^{l}}{\partial \boldsymbol{\sigma}}: \mathcal{L}: ð \boldsymbol{\varepsilon}+\left(\frac{\partial \Phi^{l}}{\partial \theta}-\frac{\partial \Phi^{l}}{\partial \boldsymbol{\sigma}}: \mathcal{L}: \boldsymbol{\alpha}\right) ð \theta
$$

Solving this system of non-linear equations and extracting the influence of each component of the strain tensor leads to:

$$
\partial \boldsymbol{\xi}=\hat{\boldsymbol{B}}^{-1}\left[\frac{\partial \boldsymbol{\Phi}}{\partial \boldsymbol{\sigma}}: \mathcal{L}: \partial \boldsymbol{\varepsilon}\right]+\hat{\boldsymbol{B}}^{-1}\left[\frac{\partial \boldsymbol{\Phi}}{\partial \theta}-\frac{\partial \boldsymbol{\Phi}}{\partial \boldsymbol{\sigma}}: \mathcal{L}: \boldsymbol{\alpha}\right] \nsim \theta
$$

which can be written in a compact form, adopting a Voigt notation with a strain vector $\tilde{\varepsilon}=\left\{\varepsilon_{11}, \varepsilon_{22}, \varepsilon_{33}, 2 \varepsilon_{12}, 2 \varepsilon_{13}, 2 \varepsilon_{23}\right\}^{T}$ :

$$
\begin{array}{r}
\partial \boldsymbol{\xi}=\mathcal{P}_{\varepsilon} \partial \boldsymbol{\varepsilon}+\mathcal{P}_{\theta} \partial \theta \\
\mathcal{P}_{\varepsilon}=\hat{\boldsymbol{B}}^{-1}\left\{\frac{\partial \boldsymbol{\Phi}}{\partial \boldsymbol{\sigma}}: \mathcal{L}\right\}, \quad \mathcal{P}_{\theta}=\hat{\boldsymbol{B}}^{-1}\left(\frac{\partial \boldsymbol{\Phi}}{\partial \theta}-\frac{\partial \boldsymbol{\Phi}}{\partial \boldsymbol{\sigma}}: \mathcal{L}: \boldsymbol{\alpha}\right)
\end{array}
$$

Note that second-order tensors and scalar quantities can be defined for the influence of strain and temperature, respectively, on a unique lead mechanism $s^{j}$ :

$$
ð \xi^{j}=\mathcal{P}_{\varepsilon}^{j} \precsim \varepsilon+\mathcal{P}_{\theta}^{j} \precsim \theta
$$

If the tangent quantities are substituted into the relation between stress and strain increments, the mechanical tangent modulus is:

$$
\begin{aligned}
& \mathrm{\partial} \boldsymbol{\sigma}=\left(\mathcal{L}-\sum_{j} \boldsymbol{\kappa}^{j} \mathcal{P}_{\varepsilon}^{j}\right) ð \varepsilon+\left(-\mathcal{L} \boldsymbol{\alpha}-\sum_{j} \boldsymbol{\kappa}^{j} \mathcal{P}_{\theta}^{j}\right) ð \theta \\
& \boldsymbol{D}^{\varepsilon}=\left(\mathcal{L}-\sum_{j} \boldsymbol{\kappa}^{j} \mathcal{P}_{\varepsilon}^{j}\right), \quad \boldsymbol{D}^{\theta}=\left(-\mathcal{L} \boldsymbol{\alpha}-\sum_{j} \boldsymbol{\kappa}^{j} \mathcal{P}_{\theta}^{j}\right),
\end{aligned}
$$




\section{Highlights}

This work presents new developments in the thermomechanical constitutive modeling of structural and functional fatigue of shape memory alloys (SMAs).

It captures the evolution of irrecoverable strain that develops during cyclic actuation of SMAs.

It describes the evolution of the structural fatigue through the evolution of an internal variable representative of damage. Final failure is predicted when such variables reaches a critical value.

The full numerical implementation of the model in an efficient scheme is described. Experimental results associated with various thermomechanical paths are compared to the analysis predictions, including fatigue structural lifetime prediction and evolution of the response during cyclic actuation.

The analysis of three-dimensional loadings paths are considered 